\title{
Review of the genus Myrabolia Reitter (Coleoptera: Cucujoidea: Myraboliidae)
}

\author{
K Wioletta Tomaszewska ${ }^{1}$ and Adam Slipinski ${ }^{2 *}$ \\ ${ }^{1}$ Museum and Institute of Zoology, Polish Academy of Sciences, Wilcza 64, 00-679 Warszawa, Poland. \\ ${ }^{2}$ CSIRO Entomology, GPO Box 1700, Canberra, ACT 2601, Australia.
}

\begin{abstract}
Species of the Australian genus Myrabolia are reviewed. Lectotypes are designated for Silvanus brevicornis Erichson, Myrabolia haroldiana Reitter, M. lindensis Blackburn and M. longicornis Blackburn. The following new species are described and illustrated: M. australis, M. blackburni, M. elongata, M. kioloa, M. lawrencei, M. micra, M. pelion and M. tasmanica. Ocholissa leai Grouvelle, originally described in Colydiidae, is here transferred to Myrabolia as M. leai (Grouvelle) comb. n. The nomenclatural history, a diagnosis and distribution data are provided for each species. A key to the species of the genus is presented.
\end{abstract}

Key words Cucujoidea, entomology, Myrabolia, Myraboliidae, review, taxonomy.

\section{INTRODUCTION}

Myrabolia Reitter is a distinctive but practically unknown group of endemic Australian beetles that have been placed in the superfamily Cucujoidea. The adult beetle strongly resembles some members of Silvaninae and this similarity was clearly guiding Reitter (1876) to place it in Cucujidae when he described this genus. Its true relationships remained unstudied until Lawrence (1988) revealed that Myrabolia and another obscure Australian taxon Tasmosalpingus Lea actually belonged to the 'primitive' cucujoid group Phloeostichidae as outlined by SenGupta and Crowson (1969). As Myrabolia could not be placed in any of the recognised subfamilies in Phloeostichidae, Lawrence and Britton (1991) established the monogeneric subfamily Myraboliinae, which was subsequently elevated to the family rank within the basal Cucujoidea (Leschen et al. 2005).

Blackburn (1892; 1903) was the only entomologist who tried to describe Australian Myrabolia and to make some sense of numerous and very externally similar species that he collected commonly over the years under bark of various eucalypts. In his 1903 paper he keyed out six species, commented upon the species described by Reitter, and erroneously synonymised Myrabolia grouvelliana Reitter with Silvanus brevicornis Erichson.

Myrabolia remained as an enigmatic and almost unknown beetle genus for about hundred years. While working on this paper we realised that Myrabolia contained a number of undescribed species. Moreover, as already stated by Blackburn, it is a very difficult group, containing species that are very similar to each other. Over the years we have accumulated rich material from many museums to assist us in preparation of a

*adam.slipinski@csiro.au comprehensive generic revision. After detailed studies of many specimens of Myrabolia and its all available types it was found that the type material of Myrabolia haroldiana Reitter included two species $-M$. haroldiana and $M$. grouvelliana Reitter and, in contradiction to Blackburn's (1903) synonymy, we propose $M$. haroldiana (not M. grouvelliana) as a junior synonym of $S$. brevicornis. In addition, we have found that Ocholissa leai, described by Grouvelle (1911) in the family Colydiidae, also belongs to Myrabolia. This transfer along with proposed new synonymies and description of eight new species brings the total number of known Myrabolia species up to 13 .

\section{MATERIALS AND METHODS}

Specimens examined were obtained from the following institutions:

ANIC, Australian National Insect Collection, Canberra

BMNH, The Natural History Museum, London

HNHM, Hungarian Natural History Museum, Budapest

MNHN, Muséum National d'Histoire Naturelle, Paris

MIZ, Museum and Institute of Zoology, PAS, Warsaw

MV, Museum of Victoria, Melbourne

NMB, Naturhistorisches Museum für Naturkunde der Humboldt Universität, Berlin

QM, Queensland Museum, Brisbane

SAM, South Australian Museum, Adelaide.

The measurements were made using a micrometer attached to a dissecting microscope as follows: (BL) body length, from apical margin of clypeus to apex of elytra; (PL) pronotal length, from the middle of anterior margin to margin of basal foramen; (PW) pronotal width at widest part; (EL) elytral length along suture, including scutellum; (EW) elytral width 

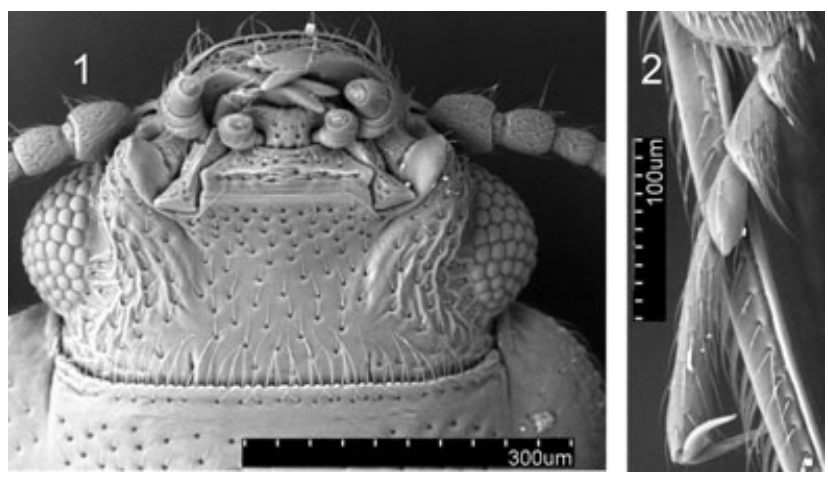

Figs 1,2. Myrabolia tasmanica: (1) head, ventral; (2) male hind tarsus.

across both elytra at widest part. Male and female genitalia were dissected, cleaned in a $10 \%$ solution of $\mathrm{KOH}$ and subsequently transferred to glycerol and drawn using a camera lucida attached to an Olympus (SZH10) dissecting microscope. After examination the genitalia were transferred to microvials and pinned beneath the specimen. The distribution maps were produced from BioLink version 2.0 (CSIRO Entomology, http://www.biolink.csiro.au). The sequence of species in the systematics section is dictated by their appearance in the key to the species.

\section{SYSTEMATICS}

\section{Myrabolia Reitter}

Myrabolia Reitter 1876: 55. Type species, by monotypy, Myrabolia haroldiana Reitter 1876 (= Silvanus brevicornis Erichson 1842: 218). - Leschen, Lawrence and Slipinski 2005: 58-60 (detailed description of adult and putative larva).

Diagnostic combination. Length $1.80-3.65$ mm. Body 2.73.4 times as long as wide, parallel-sided and flattened, feebly shiny; with distinctly seriate hairs on elytra. Winged. Head slightly longer than wide with mouthparts orientated anteriorly; eyes moderately large, coarsely facetted usually with short interfacetal setae; vertex with median stridulatory file. Antennal insertions at front of eyes, very close to clypeus, located laterally and hardly visible from above. Antenna shorter than pronotum, 11-segmented with 3-segmented club. Subantennal grooves well developed, not extending beyond eyes. Mouthparts (Fig. 1) generalised, chewing type; labrum sclerotised and partially fused to clypeus. Pronotum with anterior angles rounded; lateral edge crenulate and dentate. Prosternum long in front of coxae with finely denticulate anterior margin (Fig. 1); prosternal process broad, parallelsided between coxae, expanded apically; procoxal cavities with narrow slits exposing protrochantin, internally open, externally narrowly open. Meso-metaventral junction with metaventral knob deeply fitting into mesoventral process; mesocoxal cavities closed laterally; trochantins concealed. Elytra with punctation seriate consisting of 10 primary rows separated by secondary rows of slightly smaller punctures; punctures in rows closer to each other along basal part of elytra and more distantly separated along mid length; epipleuron narrow, complete to apex; alternate intervals often slightly elevated and with longer and closely arranged decumbent hairs. Metacoxae strongly transverse, narrowly separated. Legs long and slender; trochanterofemoral joint strongly oblique; tibiae with paired spurs; tarsi 5-5-5 in female, 5-5-4 in male (Fig. 2); tarsomeres simple. Abdomen with 5 ventrites; ventrite 1 longest, more than twice as long as ventrite 2, without postcoxal lines; usually with paired lateral pore plates in male. Ovipositor long; styli distinct and terminal; spermatheca long, slender, strongly curved, rounded at apex, with more or less distinct narrowing near point of attachment with sperm duct. Aedeagus not inverted, with articulated parameres dorsal to penis; penis with short anterior struts, narrowly rounded or acute at apex; endophallus with complex sclerites.

Distribution. Endemic to Australia with most of the species distributed along eastern and southern coast of the continent (Fig. 158).

\section{Key to the species of Myrabolia}

1 Antennomeres 4-8 at most as long as wide ......... 2

- At least antennomere 5 distinctly longer than wide .......................................... 9

2 Body black, basal part of elytra, legs and antennal club orange-brown; length 1.80-1.98 mm; pronotum less elongate, 0.74-0.77 times as long as wide; Western Australia ............................... micra sp. n.

- Body uniformly yellowish-brown to dark brown, sometimes with darker pronotum; length more than $2.00 \mathrm{~mm}$; pronotum $0.78-0.89$ times as long as wide .......................................... 3

3 Body 3.30-3.38 times as long as wide; pronotum 0.93-0.95 times as long as wide; elytra 2.00 times as long as wide; male abdominal ventrite 1 without dimorphic features; New South Wales M. elongata sp. $\mathrm{n}$.

- Body 2.76-3.09 times as long as wide; pronotum $0.83-0.93$ times as long as wide and elytra at most 1.82 times as long as wide; male dimorphic features variable ......................................... 4

4 Antennomeres 4-6 subequal in length and width; prosternal process sinuate at apex (Figs 37,46) ......... 5 Antennomere 5 at least slightly longer and wider than 4 or 6 (Figs 56,64); prosternal process widely rounded

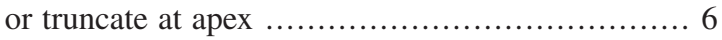

5 Body 2.76-2.91 times as long as wide; antennomere 10 distinctly broader than 9 or 11 (Figs 30,34); pronotal sides almost straight (Fig. 31); metaventral process deeply incised into mesoventrite (Fig. 37); male abdominal ventrite 1 with a pair of rounded pore plates (Fig. 36); spermatheca as in Figure 42; parameres as in Figures 40 and 41; widely distributed from Queensland to South Australia and Tasmania. M. brevicornis (Erichson) 
- $\quad$ Body 2.96-3.09 times as long as wide; antennomere 10 slightly broader than 9 or 11 (Fig. 44); pronotal sides usually weakly widening posteriorly, widest at about $1 / 3$ of basal length (Fig. 45); metaventral process shallowly incised into mesoventrite (Fig. 46); male abdominal ventrite 1 without dimorphic features; spermatheca as in Figure 54; parameres as in Figures 52 and 53; New South Wales to Tasmania and South Australia ............... M. grouvelliana Reitter Prosternal process broadly rounded at apex (Fig. 57); New South Wales ...................... M. kioloa sp. n. Prosternal process truncated at apex (Figs 65,78) ... 7

7 Body length $2.06-2.31 \mathrm{~mm}$; yellowish-brown to brown; pronotum more elongate with lateral margins weakly sinuate (Fig. 66); prosternal process between coxae about as broad as coxal diameter, and at apex 1.7-1.8 times broader than its width between coxae; elytra with sharp tooth at humeral angle (Fig. 69); [spermatheca as in Fig. 75; male abdominal ventrite 1 with obliquely oval pore plates (Fig. 68)]; Tasmania ............................. pelion sp. n. Body length 2.36-2.75 mm; dark, reddish-brown; pronotum shorter with lateral margins distinctly sinuate (Figs 77,90); prosternal process between coxae about 1.10-1.33 times broader than coxal diameter, and at apex 1.4-1.5 times broader than its width between coxae; elytra with blunt or weak humeral tooth (Figs 82,93) 8 Pronotum $0.88-0.90$ times as broad as elytra; equally wide at $1 / 4$ of apical length and at $1 / 3$ of basal length (Fig. 77); prosternal process 1.10 times as wide as coxal diameter (Fig. 78); female genitalia as in Figure 88 [male abdominal ventrite 1 with pair of oval pore plates, Fig. 81]; New South Wales, Victoria ............................. lawrencei sp. n. Pronotum 0.87 times as wide as elytra; widest at about $1 / 3$ of basal length (Fig. 90); prosternal process between coxae 1.33 times wider than coxal diameter (Fig. 92); female genitalia and shape of spermatheca as in Figure 95 [male unknown]; Tasmania . M. australis sp. $\mathrm{n}$. Body 3.06-3.16 times as long as wide, chestnut or yellowish-brown; antennomeres 7-8 longer than wide (Fig. 98); elytra 1.86-1.90 times longer than wide; prosternal process rounded at apex (Fig. 99); male abdominal ventrite 1 with moderately large, obliquely oval pore plates (Fig. 103); Victoria ............................ M. longicornis Blackburn Body 2.84-3.05 times as long as wide, dark yellowishbrown or bicoloured (brown and black); antennomeres $7-8$ at most as long as wide (Figs 111,122,137); elytra 1.68-1.84 times longer than wide; prosternal process truncate or sinuate at apex; male abdominal ventrite 1 with pore plates differently shaped (Figs 115,123)

Among antennomeres 3-8 only antennomere 5 distinctly longer than wide (Fig. 111); pronotum 0.76-
0.79 times as long as wide and elytra $1.68-1.71$ times as long as wide; mesoventral process with very coarse punctures almost touching each others (Fig. 110); tegmen with very long parameres (Figs 118,119); [male abdominal ventrite 1 with small pore plates as in Fig. 115]; SA ..................... M. blackburni sp. n.

- Antennomeres 3-5 longer than wide (Figs 122,137,146); pronotum 0.79-0.88 times as long as wide and elytra 1.71-1.84 times as long as wide; meso-metaventral process with moderately coarse punctures 0.5-2 diameters apart (Figs 125,136,148); tegmen with parameres moderately long ........... 11

11 Body uniformly brown; prosternal process sinuate at apex (Fig. 125); mesoventral process with punctures 0.5-1.0 diameter apart (Fig. 125); male abdominal ventrite 1 with a pair of very small, oval, obliquely directed pits (Fig. 124); SA, Victoria .. M. lindensis Blackburn Body bicoloured (black and brown); prosternal process truncate at apex; mesoventral process with punctures 1.0-2.0 diameters apart (Figs 136,148); male abdominal ventrite 1 with a pair of moderately large or large pore plates (Figs 139,152) ...................... 12

12 Elytra 1.71-1.80 times as long as wide; pronotum $0.38-0.39$ times as long as elytra and $0.83-0.86$ times as wide as elytra; abdominal pore plates moderately large, rounded (Fig. 139); spermatheca as in Figure 144; parameres as in Figures 142 and 143; New South Wales, Victoria, Tasmania M. leai (Grouvelle)

- Elytra 1.80-1.84 times as long as wide; pronotum $0.40-0.42$ times as long as elytra and 0.87-0.89 times as wide as elytra; pore plates very large, oval, setose (Fig. 152); spermatheca as in Figure 157; parameres as in Figures 155 and 156; Tasmania M. tasmanica sp. n.

\section{Myrabolia micra sp. n. (Figs 3-15, I58)}

Types. Holotype, male. Western Australia. 'Shannon, Shannon R. 3 Jan. 1986, WA, C. Reid, on Agonis flexuosa' (ANIC). Paratypes: same data as holotype (3: ANIC; 2: MIZ). Diagnosis. The small size of this species combined with its black colour, except for orange elytral bases, and relatively transverse pronotum separate it easily from all of its congeners. It is so far the only species endemic to Western Australia. Description. Length $1.80-1.98 \mathrm{~mm} ; \mathrm{BL} / \mathrm{EW}=2.77-2.88$; $\mathrm{PL} / \mathrm{PW}=0.74-0.77 ; \quad \mathrm{PL} / \mathrm{EL}=0.37-0.39 ; \quad \mathrm{PW} / \mathrm{EW}=0.88-$ $0.89 ; \mathrm{EL} / \mathrm{EW}=1.69-1.76 ; \mathrm{EL} / \mathrm{PL}=2.50-2.60$.

Body elongate-oval (Fig. 3), shiny; almost black with basal part of elytra, legs and antennal club orange; covered with long hairs. Antenna (Fig. 4) 0.9 times as long as pronotum, antennomere 3 at least slightly longer than broad and distinctly longer than antennomere 4; antennomeres 4-8 at most as long as wide; antennomere 5 slightly wider than 4 or 6 ; club moderately long and broad, rather compact. Pronotal sides weakly sinuate (Fig. 7), widest at about $1 / 3$ of basal length; lateral margins crenulate with small, sharp denticles; posterior angle 

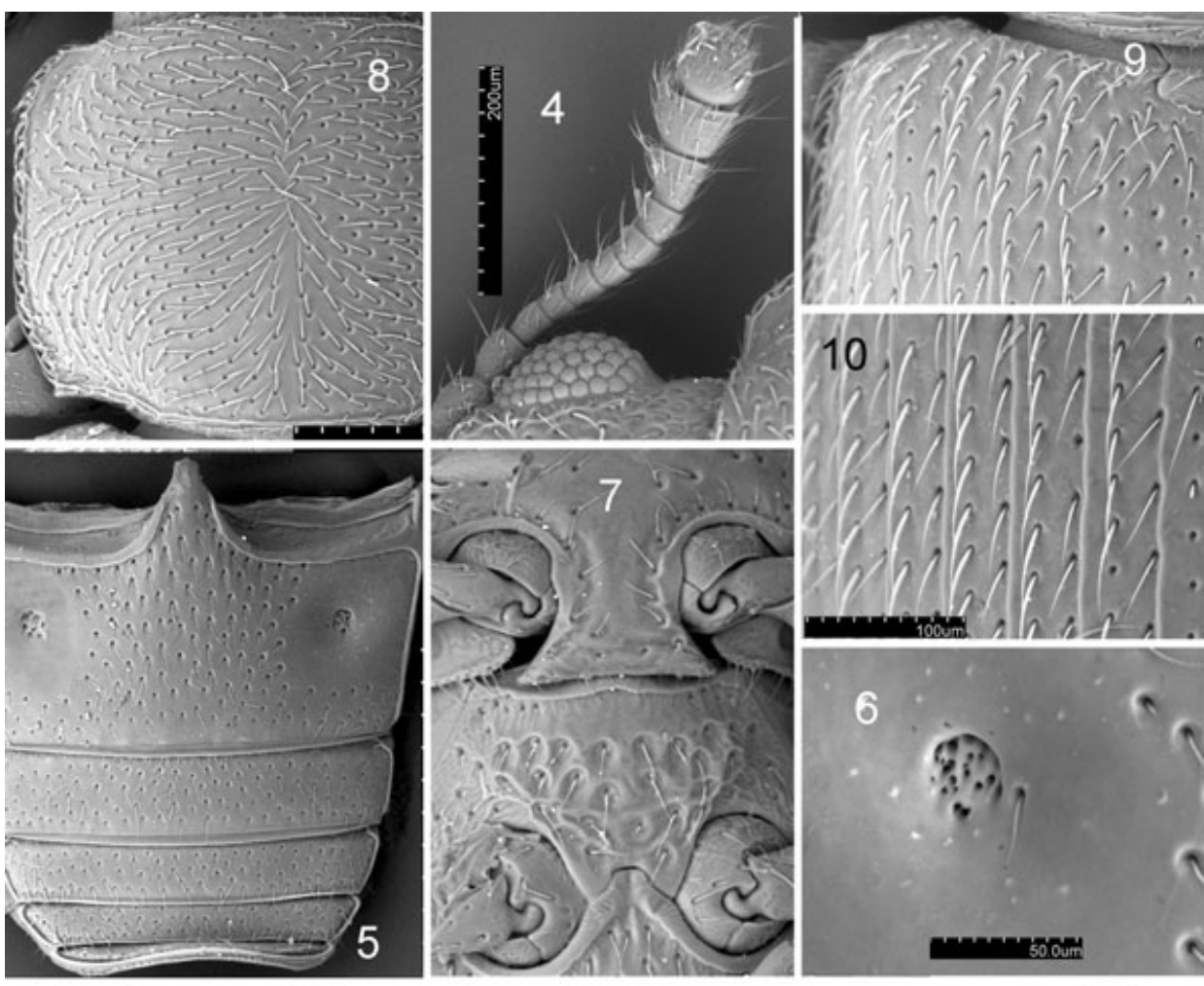

Figs 3-15. Myrabolia micra: (3) dorsal view; (4) antenna; (5) male abdomen; (6) male abdominal ventrite 1, lateral part; (7) pro- and mesothorax, ventral and metaventral junction; (8) pronotum; (9) elytron, basal part; (10) elytron, disc; (11) sternite of male genital segment, ventral view; (12) median lobe; (13) tegmen, ventral; (14) tegmen, lateral; (15) female genitalia, ventral.
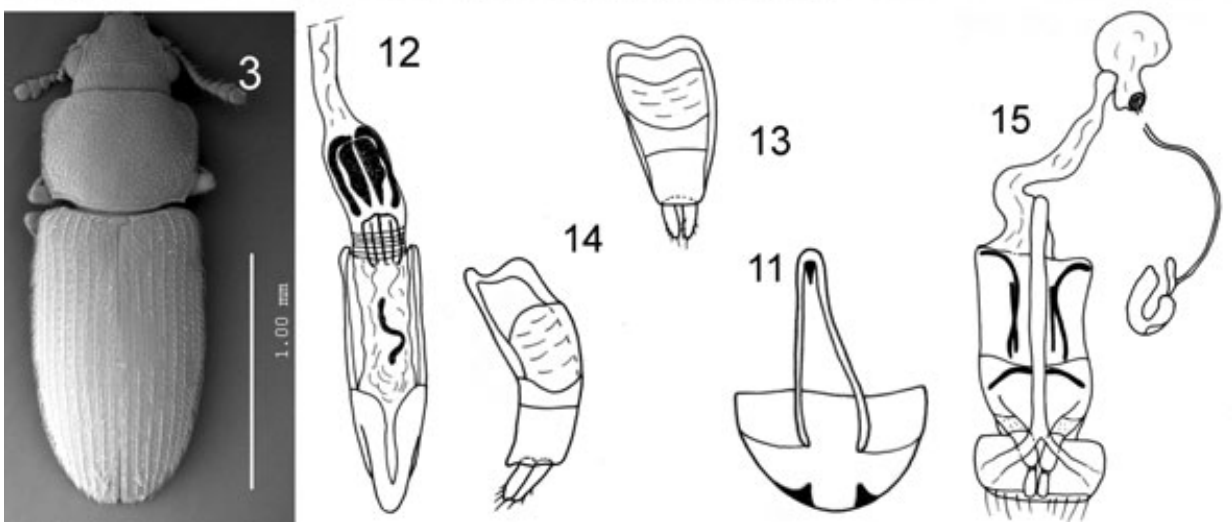

with weak tooth; punctures moderately large and dense, about 2 diameters apart laterally and 2.5-3.0 diameters apart on disc. Prosternal process (Fig. 7) 1.2 times as wide as coxal diameter and at apex 1.55 times broader than its width between coxae; apex truncate to weakly arcuately concave; sparsely punctured. Elytra (Figs 3,9,10) with punctures in rows 2.0-3.0 diameters apart; lateral margins along shoulders rather sharply crenulate, with tooth at anterior angle as large as teeth below it. Meso-metaventral junction coarsely punctured, punctures $0.5-$ 1.0 diameter apart, metaventral part widely bordered and deeply incised into mesoventrite (Fig. 7). Male abdominal ventrite 1 with small, round porous plate (Figs 5,6). Sternite of male genital segment and median lobe as in Figures 11 and 12. Parameres subcylindrical, almost 1/4 as long as rest of tegmen, with long apical and few short, subapical setae (Figs 13,14). Female genitalia as in Figure 15.

Etymology. The name of this species is an arbitrary combination of letters referring to the small body of this species.
Distribution. Southern part of Western Australia (Fig. 158).

\section{Myrabolia elongata sp. n. ( Figs I6-27, I58)}

Types. Holotype, male. New South Wales. '35.30S 150.18E Kioloa SF, 15 km NE Batemans Bay, NSW, III. 1987. M.G. Robinson, ex sticky trap' (ANIC). Paratypes, females: same data as holotype but Feb.87, flight intercept trap (1: MIZ). Australian Capital Territory. (35.22S 148.50E) Blundells Ck. $3 \mathrm{~km}$ E of Piccadilly Circus, 850 m, ACT, May 1984, Weir, Lawrence, Johnson/flight intercept window, through trap (1: ANIC).

Diagnosis. This species is characterised by its parallel-sided, narrow, elongate body with pronotum almost as wide as elytra. Description. Length 2.36-2.83 mm; BL/EW = 3.30-3.38; $\mathrm{PL} / \mathrm{PW}=0.93-0.95 ; \quad \mathrm{PL} / \mathrm{EL}=0.43-0.45 ; \quad \mathrm{PW} / \mathrm{EW}=0.94-$ $0.95 ; \mathrm{EL} / \mathrm{EW}=2.00 ; \mathrm{EL} / \mathrm{PL}=2.30-2.34$.

Body strongly elongate, uniformly dark brown, covered with long hairs. Antenna (Fig. 16) 0.75 times as long as prono- 

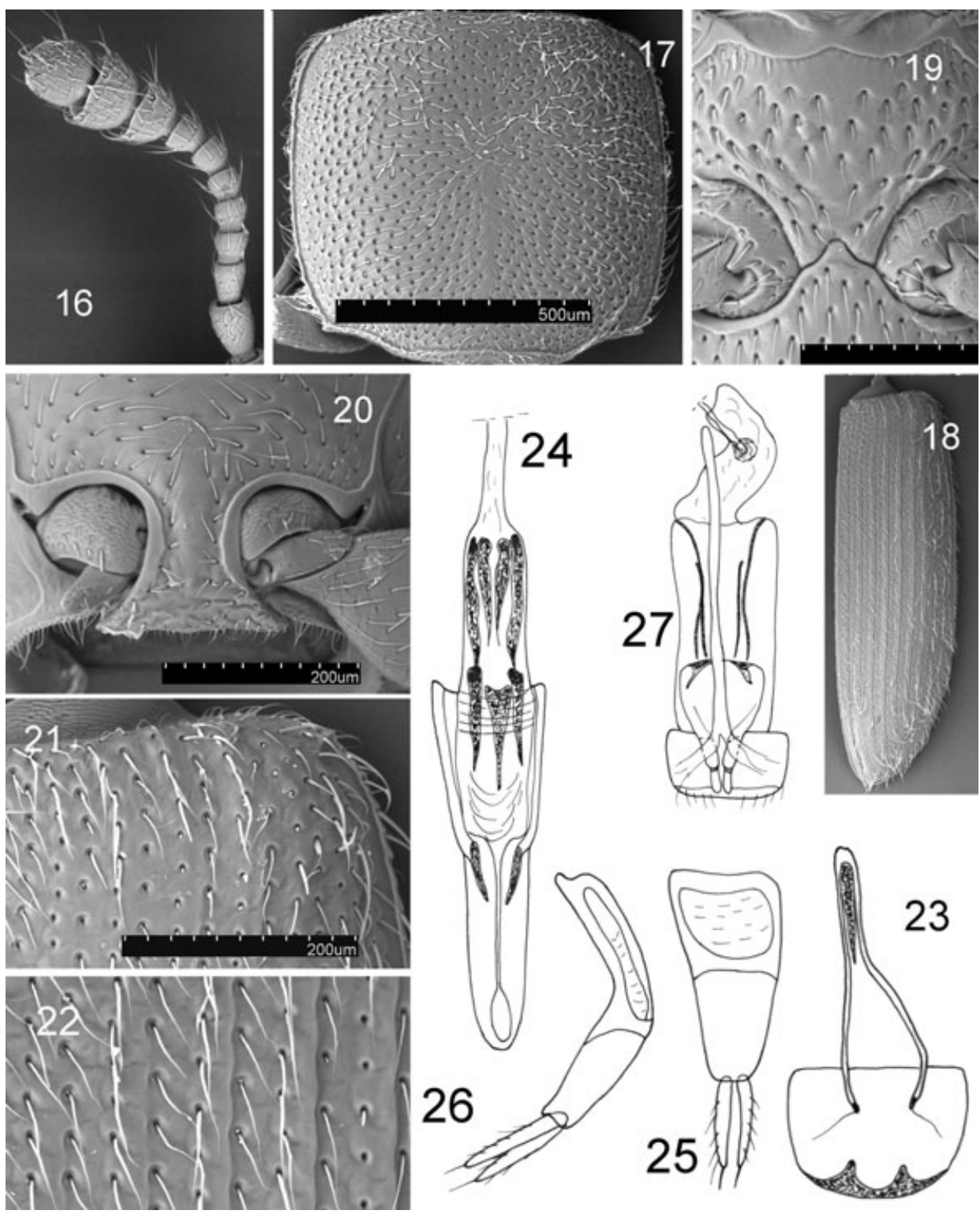

Figs 16-27. Myrabolia elongata: (16) antenna; (17) pronotum; (18) elytron, right, dorsal; (19) mesothorax, ventral and metaventral junction; (20) prosternal process; (21) elytron, basal part; (22) elytron, disc; (23) sternite of male genital segment, ventral view; (24) median lobe; (25) tegmen, ventral; (26) tegmen, lateral; (27) female genitalia, ventral. tum; antennomere 3 at least slightly longer than wide and distinctly longer than antennomere 4; antennomeres 4-8 at most as long as wide; club long, broad and moderately compact. Pronotal sides almost straight (Fig. 17), parallel, weakly rounded towards anterior and posterior angles; lateral margins very finely crenulate, with very small denticles, larger along basal half; posterior angle with weak tooth; punctures moderately large, 0.5-1.0 diameter apart laterally and 2-3 diameters apart on disc. Prosternal process (Fig. 20) 0.85 times as wide as coxal diameter, strongly expanded laterally at apex; at apex 2.1 times its width between coxae; apical edge weakly sinuate. Elytra (Figs 18,21,22) with punctures in rows 1.5-2.0 diameters apart; lateral margins along shoulders finely crenulate, with small tooth at anterior angle. Meso-metaventral junction covered with punctures 1.5-2.5 diameter apart (Fig. 19); metaventrite not bordered, narrowly but rather deeply incised into mesoventrite (Fig. 19). Male abdominal ventrite 1 simple, without dimorphic features. Sternite of male genital segment and median lobe as in Figures 23 and 24. Tegmen with parameres subcylindrical, very long, almost 1:2.5 as long as rest of tegmen, densely setose (Figs 25,26). Female genitalia as in Figure 27 (spermatheca not observed). Etymology. The name of this species refers to its strongly elongate body.

Distribution. New South Wales (Fig. 158).

\section{Myrabolia brevicornis (Erichson) (Figs 28-42, I58)}

Silvanus brevicornis Erichson 1842: 218.

Myrabolia brevicornis: Blackburn 1903: 151.

Myrabolia haroldiana Reitter 1876: 56. Syn. n.

Types. Lectotype male of Silvanus brevicornis, Terra v. Diem, Schayer/brevicornis Er./Silvanus Er./Myrabolia brevicornis (Er.) D.G.H. Halstead, det. 1992/Syntype Silvanus brevicornis (Er.) D.G.H. Halstead, det. 1992/Zool. Mus. Berlin/53366 (NMB); paralectotypes, same data as lectotype 
Figs 28-37. Myrabolia brevicornis: (28) dorsal view; (29) ventral view; (30) antenna; (31) pronotum; (32) elytron, basal part; (33) elytron, disc; (34) antennal club; (35) male abdomen; (36) male abdominal ventrite 1, lateral part; (37) pro- and mesothorax, ventral and metaventral junction.
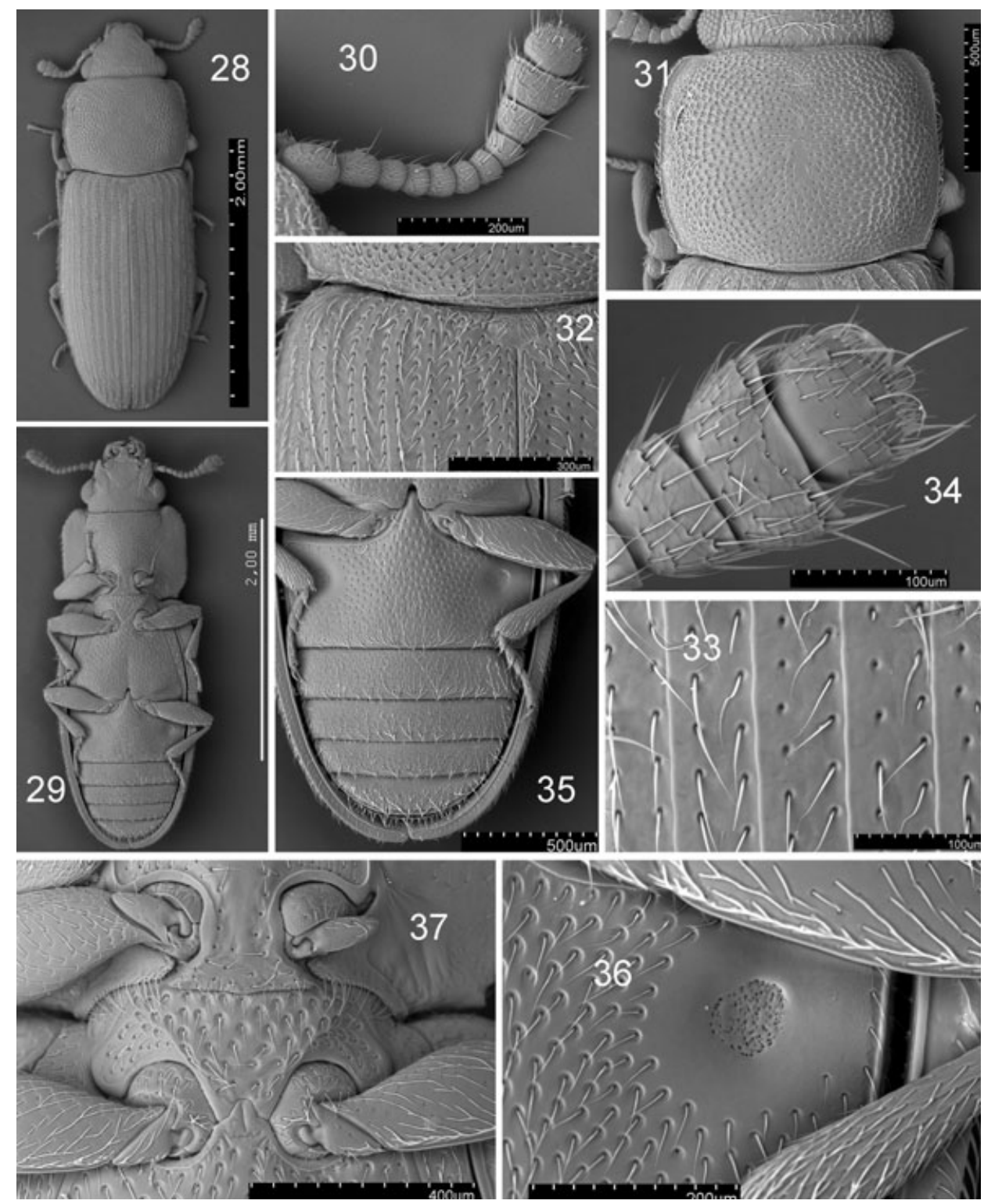
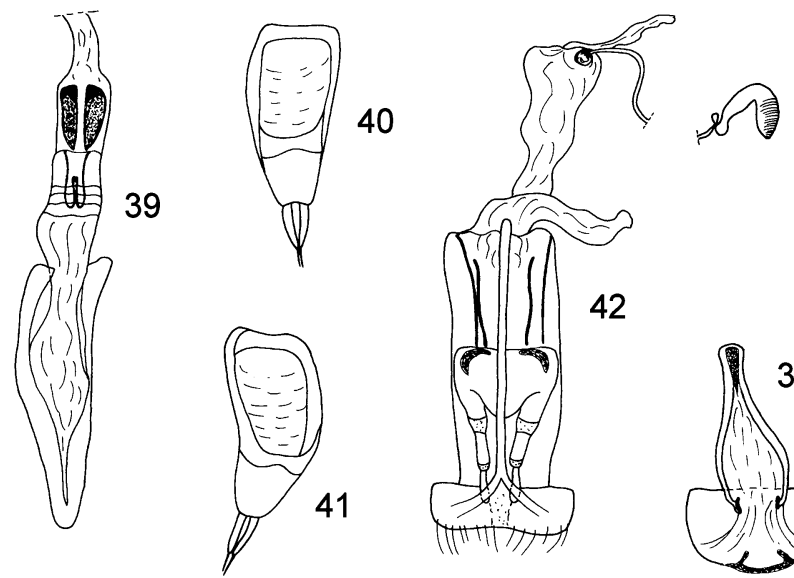

Figs 38-42. Myrabolia brevicornis: (38) sternite of male genital segment, ventral view; (39) median lobe; (40) tegmen, ventral; (41) tegmen, lateral;

(42) female genitalia, ventral.
(3: NMB). Lectotype male of Myrabolia haroldiana, Australia, Rockhampton, H. Deyrolle/Myrabolia (m Sylvanidae) haroldiana m./Museum Paris (MNHN); paralectotype, female, same data as lectotype (MNHN). Present designation. The lectotypes of Silvanus brevicornis Erichson 1842 and Myrabolia haroldiana Reitter 1876 are here designated to fix the taxonomic status of these species.

Other specimens examined. Queensland. Mt Coot-tha (27.29S 152.57E), 17.xii.1990, J.F. Lawrence, surfaces at night (1: ANIC); Bundaberg, 1904, R.C.L. Perkins (1: BMNH); 3.5 mls. Kuranda-Mareeba Rd, 20.i.1962, E.B. Britton (1: BMNH). New South Wales. Armidale, i.1934, F.E. Wilson (1: MV); Sydney, Lea (7; SAM). Australian Capital Territory. Blundells Cr Rd, $850 \mathrm{~m}, 3.5 \mathrm{~km}$ E of Piccadilly Circus, 6.ii.1983, J.F. Lawrence; in bark of living Eucalyptus (1: ANIC); same but 18.xi.1984 (1: MIZ, totally dissected); Black Mt (35.17S 149.07E) near light trap, 23.xi.-31.xii.1988; J. Lawrence, A. Calder, M. Hansen, Berlesate, ANIC 1089, 
wood \& bark from standing Eucalyptus (2: ANIC). Victoria. Grampians, x.1928, F.E. Wilson (1: ANIC); Black Rock, VII.29 (2: BMNH); Glenferrie, K. Guichard (3: ANIC); Mt Buffalo, Blackburn (1: SAM); Emerald (1: SAM); Frankston (1: SAM). South Australia. Adelaide (15: SAM); Mt Lofty (2: SAM); Murray River, R. Burton (2: SAM); Brookfield Con. Park (34.19S 139.32E), 3-12.ix.1991, T. Weir \& W. Dressler, under bark (5: ANIC); 10 km SW Strathalbyn, xii.1974, under Eucalyptus bark, P. McQuillan (1, SAM). Tasmania. Snug, 22.xi.1981, Bornemissza (2: MIZ; 2: HNHM); Hobart, Seven Mile Beach, 20.ix.1981 (2: HNHM; 1 totally dissected MIZ); Hobart, Mt. Nelson, i.1983, Bornemissza (2: HNHM); Bruny Island 7.vi.1984, G.F. Bornemissza (2: HNHM); Hobart, J.J. Walker (4: BMNH; 2: MIZ); New Norfolk, J.J. Walker, G.C. (1: BMNH); Granton, 5.ii.1983, Bornemissza (1: HNHM); Launceston (20: SAM).

Diagnosis. Myrabolia brevicornis resembles $M$. grouvelliana most closely, especially in body shape and colouration but $M$. brevicornis is shorter, less elongate and the antennal club short and compact with antennomere 10 distinctly wider than 9 and 11. Moreover abdominal ventrite 1 in male of $M$. grouvelliana lacks a pair of lateral pore plates which are distinct and deep in M. brevicornis.

Description. Length $2.85-3.65 \mathrm{~mm} ; \mathrm{BL} / \mathrm{EW}=2.76-2.91$; $\mathrm{PL} / \mathrm{PW}=0.82-0.87 ; \quad \mathrm{PL} / \mathrm{EL}=0.42-0.46 ; \quad \mathrm{PW} / \mathrm{EW}=0.85-$ $0.89 ; \mathrm{EL} / \mathrm{EW}=1.63-1.73 ; \mathrm{EL} / \mathrm{PL}=2.17-2.36$.

Body (Figs 28,29) elongate-oval, uniformly dark brown, covered with dense and rather long hairs. Antenna (Figs 30,34) 0.7 or less times as long as pronotum with antennomeres 4-8 at most as long as wide; antennomere 3 slightly longer than wide; club moderately long, wide and compact with antennomere 10 distinctly wider that 9 and 11. Pronotal (Fig. 31) sides almost straight, lateral margins rather weakly crenulate, with small denticles; posterior angle with weak tooth; punctures large and dense, almost touching each others laterally and 1-2 diameters apart on disc. Prosternal process (Fig. 37) about 1.15 times as wide as coxal diameter, strongly expanded apically, at apex 1.8 times its width between coxae; apical edge weakly sinuate. Elytra (Figs 28,32,33) with punctures in rows 1.0-2.0 diameters apart; lateral margins along shoulders very finely crenulate. Meso-metaventral junction covered with punctures 1.0-1.5 diameter apart (Fig. 37), metaventrite deeply incised into mesoventrite (Fig. 37). Abdominal ventrite 1 in male with moderately large and deep, round pore plate on each side (Figs 35,36). Sternite of male genital segment and median lobe as in Figures 38 and 39. Tegmen with parameres cylindrical, 1/4 as long as rest of tegmen, with long apical setae (Figs 40,41). Female genitalia as in Figure 42.

Distribution. Widely distributed from Tasmania through Victoria and New South Wales to northern Queensland (Fig. 158).

\section{Myrabolia grouvelliana Reitter (Figs 43-54, I 58)}

Myrabolia grouvelliana Reitter 1878: 179.

Myrabolia longicollis Blackburn 1903: 149. Syn. n.

Myrabolia parva Blackburn 1892: 32. Syn. n.

Types. Holotype of Myrabolia grouvelliana, female. Tasmania, Deyrolle/Myrabolia grouvelliana m./Museum Paris
(MNHN); Myrabolia n.g. haroldiana m., Australia, Deyrolle, Type Reitter, Museum Paris (2: MNHN; syntypes of haroldiana). Holotype of Myrabolia longicollis, Victoria/Type, HT/Myrabolia longicollis Blackburn/Australia, Blackb. Coll, B.M. 1910-236 (BMNH). Holotype of Myrabolia parva, female, 'Myrabolia parva Blackb./type, H.T./Australia Blackburn coll. 1910-236/Narberhong, Healesville, 30-II-30/T 4141, Sydney' (BMNH).

Other specimens examined. New South Wales. K.K. Spence (1: ANIC); Mt Victoria, Biro 1900 (3:ANIC); coll. Kessel (1: MIZ); Nowra (34.53S 150.36E), $119 \mathrm{~km} \mathrm{~S}$ of on NerrigaNowra rd. 19.i.71, woodlands, S. Misko, K. Pullen (2: ANIC; 1: MIZ); same but (35.08S 150.02E) (1: ANIC); Round Hill: near Lake Cargelligo, 11.i.1981, Hangay \& Vojnits (2: HNHM); Muswellbrook, E.W. Ferguson (1: ANIC; 1: MIZ); Wollombi Falls, 40 km E Armidale, 1978-1979 R. Noske (1: ANIC). Australian Capital Territory. Black Mt, light trap, 6.ii.68, M.S. Upton (1: ANIC); same but 1.ii.68 (1: ANIC); Piccadilly Circ. Brindabella Rng., 7.xi.1982, Eucalyptus bark, J. Lawrence (1: ANIC); Victoria. Healesville (1: BMNH); Coranderrk Res. Healesville, iii.1979, P. Gullan, under Eucalyptus bark (1: ANIC). South Australia. Adelaide, Hart, 1886 (5: BMNH); A.H. Elston (1: ANIC); Mt Magnificent NP, Southern Mt Lofty Range, 1.iii.1974, P. McQuillan (4; SAM); 2 mi NE of Lenswood, P. McQuillan \& P. March (3: SAM). Tasmania. Liffey Falls State Reserve, Lower Picnic Area (41.41S 146.45E), 9.xii.1993, D.S. Horning, Jr, under bark live Eucalyptus sp. (1: ANIC); Hobart, Seven Mile Beach, Bornemissza, 20.ix.1981 (2: HNHM); Granton, 5.ii.1983, Bornemissza (2: HNHM); Hobart, Lea (1: SAM); Bruny Island 7.vi.1984, G.F. Bornemissza (9: HNHM; 5: MIZ); Hobart, J.J. Walker (2: BMNH). Sandy Is., under bark, F.E. Wilson (3: VM).

Diagnosis. This species is most similar to M. brevicornis, but it can be distinguished from that species by more elongate body, the antennomere 10 not wider than 9 or 11 , the male abdominal ventrite 1 simple, the pronotum with lateral sides slightly widened backwards, widest at about $1 / 3$ of basal length and more elongate elytra.

Description. Length 2.35-3.23 mm; $\mathrm{BL} / \mathrm{EW}=2.96-3.08$; $\mathrm{PL} / \mathrm{PW}=0.83-0.88 ; \quad \mathrm{PL} / \mathrm{EL}=0.42-0.44 ; \quad \mathrm{PW} / \mathrm{EW}=0.87-$ $0.92 ; \mathrm{EL} / \mathrm{EW}=1.73-1.82 ; \mathrm{EL} / \mathrm{PL}=2.30-2.46$.

Body (Fig. 43) long-oval, uniformly dark brown, covered with long hairs. Antenna (Fig. 44) 0.85 times as long as pronotum with antennomere 3 at least slightly longer than broad and distinctly longer than antennomere 4 ; antennomeres 4-8 at most as long as wide; club moderately long and broad, and rather compact. Pronotal sides (Fig. 45) parallel, weakly widening towards base; widest at about $1 / 3$ of basal length; lateral margins weakly to distinctly crenulate, with small denticles; posterior angle with weak tooth; punctures large and dense, laterally about 0.5 diameter apart to almost touching each other and 2-3 diameters apart on disc. Prosternal process (Fig. 46) about 1.1 times as wide as coxal diameter, strongly expanded apically, at apex 1.65-1.73 times its width between coxae; apical edge weakly to distinctly sinuate. Elytra (Figs 43,48,49) with punctures in rows 2.0-3.0 diameter apart; 

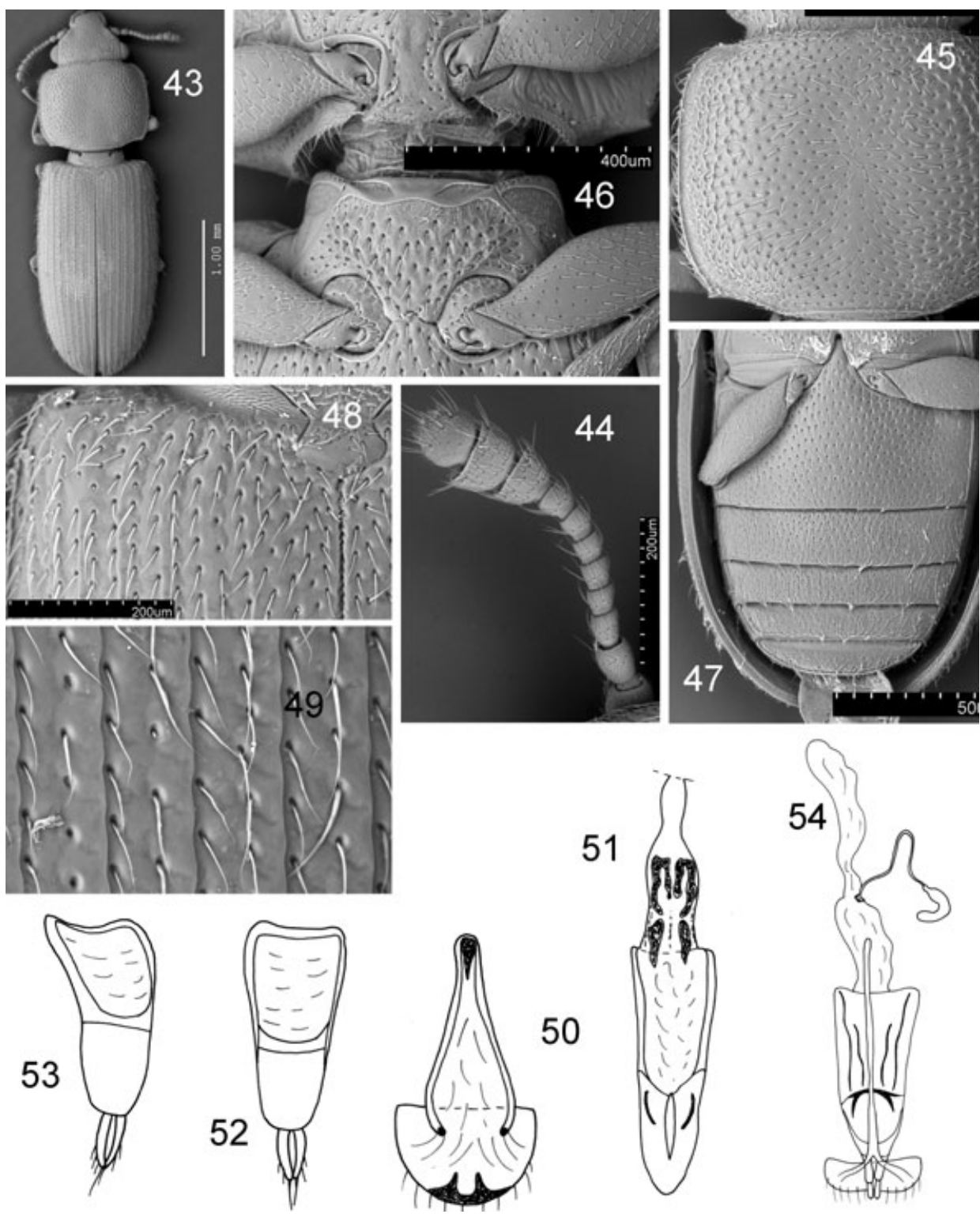

Figs 43-54. Myrabolia grouvelliana: (43) dorsal view; (44) antenna; (45) pronotum; (46) pro- and mesothorax, ventral and metaventral junction; (47) male abdomen; (48) elytron, (50) sternite of male genital segment, ventral view; (51) median lobe; (52) tegmen, ventral; (53) tegmen, lateral; (54) female genitalia, ventral. basal part; (49) elytron, disc;

antennal club stouter than in other populations. However, both male and female genitalia have not shown sufficient differences justifying description of a new species. apart (Fig. 46); metaventrite not bordered, shallowly incised into mesoventrite (Fig. 46). Male abdominal ventrite 1 simple, without dimorphic features (Fig. 47). Sternite of male genital segment and median lobe as in Figures 50 and 51. Tegmen with parameres cylindrical, curved outwardly, almost $1 / 3$ as long as rest of tegmen, with long apical and few short subapical setae (Figs 52,53). Female genitalia as in Figure 54.

Distribution. New South Wales, Victoria, South Australia and Tasmania (Fig. 158).

Discussion. It appears that individual variation in M. grouvelliana is much more apparent than in other species of the genus. Among specimens studied there were profound differences in their body size, the shape of pronotum, length of antennomeres, etc. The most distinct specimens were from the Brunny Island, with pronotal sides almost straight, parallelsided, prosternal process distinctly sinuate apically and the

\section{Myrabolia kioloa sp. n. (Figs 55-62, 158)}

Types. Holotype, female. New South Wales. '35.30S 150.18E Kioloa SF, 15 km NE Batemans Bay, NSW, III. 1987. M.G. Robinson, flight intercept trap (ANIC). Paratype, female: same data as holotype (1: MIZ).

Diagnosis. This species is most similar to Myrabolia pelion but is distinguished by the larger body, more elongate pronotum and the apex of the prosternal process rounded and less expanded apically.

Description. Length $\quad 2.46-2.60 \mathrm{~mm} ; \quad \mathrm{BL} / \mathrm{EW}=2.90-3.09$; $\mathrm{PL} / \mathrm{PW}=0.83-0.89 ; \quad \mathrm{PL} / \mathrm{EL}=0.41-0.43 ; \quad \mathrm{PW} / \mathrm{EW}=0.84$ $0.87 ; \mathrm{EL} / \mathrm{EW}=1.70-1.78 ; \mathrm{EL} / \mathrm{PL}=2.34-2.46$. 


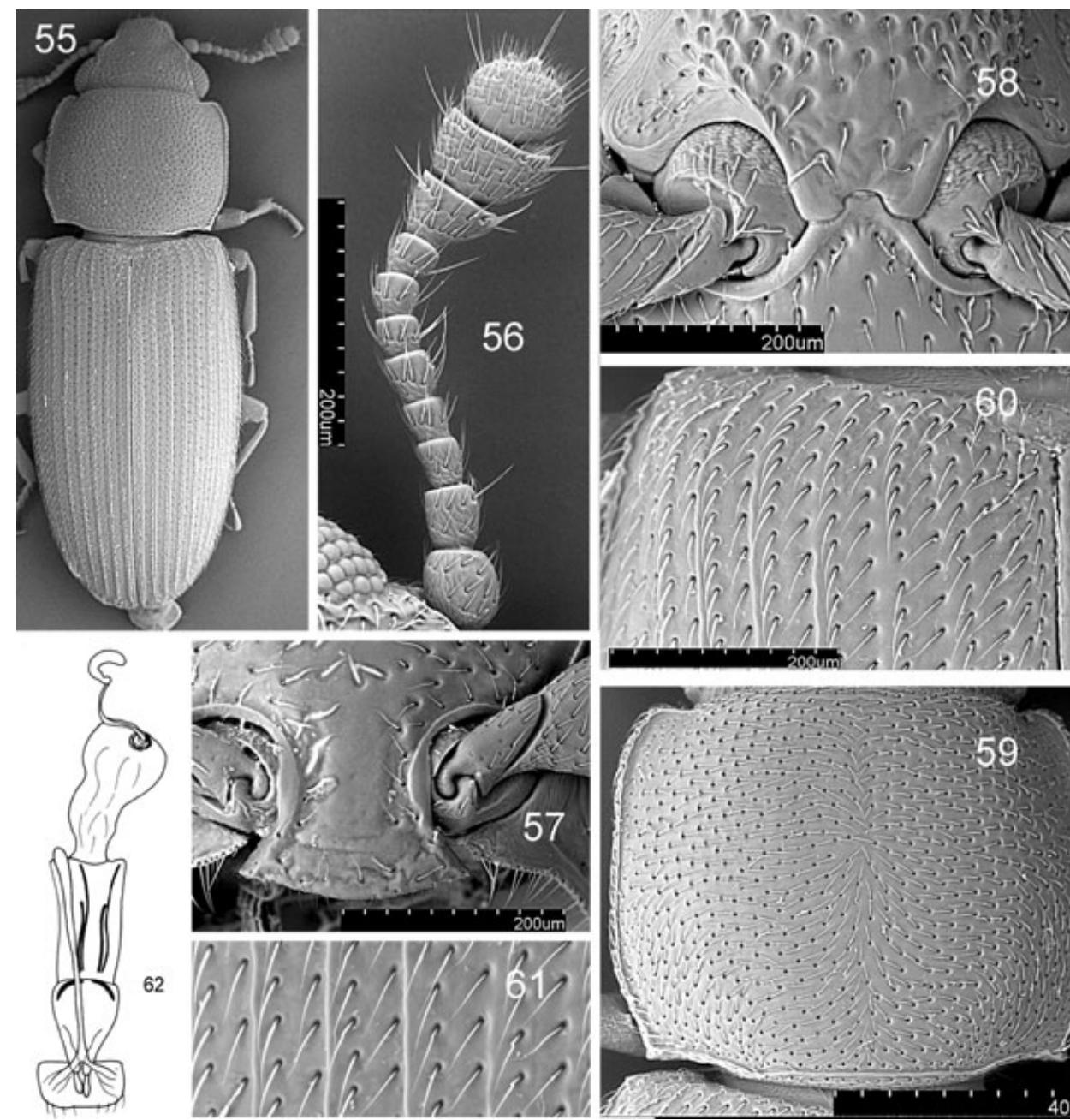

Figs 55-62. Myrabolia kioloa: (55) dorsal view; (56) antenna; (57) prosternal process; (58) mesothorax, ventral and metaventral junction; (59) pronotum; (60) elytron, basal part; (61) elytron, disc; (62) female genitalia, ventral.
Body (Fig. 55) long-oval, uniformly light to dark brown, covered with long hairs. Antenna (Fig. 56) 0.9 times as long as pronotum with antennomeres 4 and 6-8 at most as long as wide; antennomeres 3 and 5 slightly longer than wide and equal in length; club long, broad and compact. Pronotal (Fig. 59) sides weakly sinuate; broadest at about $1 / 3$ of basal length; lateral margins weakly crenulate, with small denticles; posterior angle with small tooth; anterior angles somewhat produced anteriorly; punctures moderately large, 1-1.5 diameters apart laterally and 2-2.5 diameters apart on disc. Prosternal process (Fig. 57) sparsely punctured only along lateral and apical margins; 1.4 times as wide as coxal diameter and moderately expanded apically, at apex 1.5 times its width between coxae; rounded apically. Elytra (Figs 55,60,61) with punctures in rows 1.5-2.5 diameters apart; crenulate and distinctly dentate along shoulders. Mesometaventral junction covered with punctures 1.5-2.0 diameters apart (Fig. 58); metaventrite narrowly bordered and broadly but rather shallowly incised into mesoventrite (Fig. 58). Female genitalia as in Figure 62. Male unknown.
Etymology. The species name is formed from its type locality at the Kioloa State Forest.

Distribution. New South Wales (Fig. 158).

\section{Myrabolia pelion sp. n. (Figs 63-75, I58)}

Types. Holotype, male. Tasmania. '41.52S 146.03E Tas., Pelion Gap 2 km ENE Mt. Ossa 1120 m, 13 Feb.-7 March 1991, I. Naumann, M. Horak, Malaise \#6/Eucalyptus coccifera woodland with alpine heath m\#6/ANIC database no. 25 030019' (ANIC); Paratypes: Pelion Hut (41.50S 146.03E), 3 km S Mt. Oakleigh, 860 m, 13.ii.-7.iii.1991, I. Naumann, M. Horak, Malaise \#1 closed forest/ANIC database no. 25030019 (1: MIZ); Mt. Barrow Rd 890 m, 15-17.ii.1980, Nothofagus, etc. A. Newton, M. Thayer/Pyrethrum fogging, Melaleuca bark (1: ANIC); Bathurst Harbour (43.25S 146.10E), 25-29.xi.1991, Melaleuca, I. Naumann, G. Clarke, heathy sedge land \& closed forest margin/ANIC database no. 25 030024 (1: MIZ); same but 18-21.ii.1991, A. Calder, W. Dressler, sweeping button grass/ANIC database no. 25030020 (1: ANIC); Hobart, Lea (1: SAM); Hob. Lea. 10560, Myrabolia longicornis, Bl. (1: SAM). 
Figs 63-75. Myrabolia pelion: (63) dorsal view; (64) antenna; (65) pro- and mesothorax, ventral and metaventral junction; (66) pronotum; (67) male abdomen; (68) male abdominal ventrite 1 , lateral part; (69) elytron, basal part; (70) elytron, disc; (71) sternite of male genital segment, ventral view; (72) median lobe; (73) tegmen, ventral; (74) tegmen, lateral; (75) female genitalia, ventral.
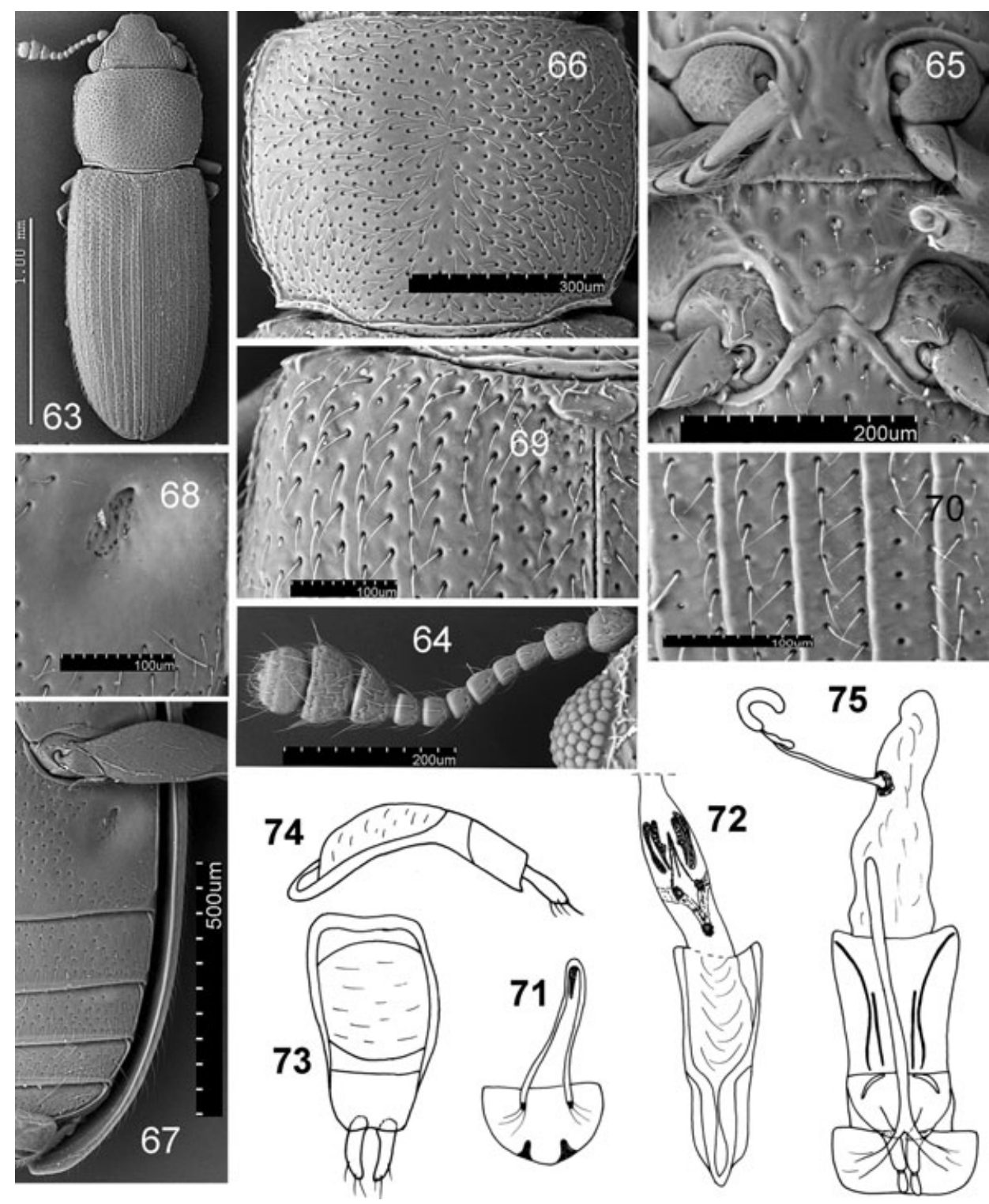

Diagnosis. Myrabolia pelion closely resembles $M$. kioloa, but can be distinguished from it by its smaller size, the pronotum less elongate and apex of the prosternal process truncate and more expanded laterally.

Description. Length 2.06-2.31 mm. BL/EW = 2.90-3.06; $\mathrm{PL} / \mathrm{PW}=0.82-0.85 ; \quad \mathrm{PL} / \mathrm{EL}=0.40-0.42 ; \quad \mathrm{PW} / \mathrm{EW}=0.86-$ $0.90 ; \mathrm{EL} / \mathrm{EW}=1.74-1.82 ; \mathrm{EL} / \mathrm{PL}=0.2 .34-2.50$.

Body (Fig. 63) long-oval, uniformly light to dark brown, covered with long hairs. Antenna (Fig. 64) nearly as long as pronotum with antennomeres 4 and 6-8 at most as long as wide; antennomeres 3 and 5 at least slightly longer than broad; antennomere 5 slightly longer and wider than 4 or 6 ; club long, broad and rather compact. Pronotum (Fig. 66) with sides weakly sinuate; broadest at about $1 / 3$ of basal length; lateral margins crenulate, with sharp denticles; posterior angle with small, sharp tooth; anterior angles rounded; punctures moderately large, 1.5-2 diameters apart (only in middle line 2-2.5 diameters apart). Prosternal process
(Fig. 65) very sparsely and finely punctured, 1.1 times as wide as coxal diameter, strongly expanded apically, at apex 1.7-1.8 times its width between coxae, apical margin truncate. Elytra (Figs 63,69,70) with punctures in rows 2-3 diameters apart; lateral margins along shoulders finely crenulate, with sharp tooth at anterior angle. Meso-metaventral junction covered with punctures 1-1.5 diameters apart (Fig. 65); metaventrite narrowly bordered, widely but rather shallowly incised into mesoventrite (Fig. 65). Male abdominal ventrite 1 with a pair of rather small, obliquely oval pore plates (Figs 67,68). Sternite of male genital segment and median lobe as in Figures 71 and 72 . Tegmen with parameres somewhat curved outwardy, about $1 / 4$ as long as rest of tegmen (Figs 73,74). Female genitalia and shape of spermatheca as in Figure 75.

Etymology. The name of this species is formed from Pelion Gap, the locality where the holotype was collected.

Distribution. Tasmania (Fig. 158). 

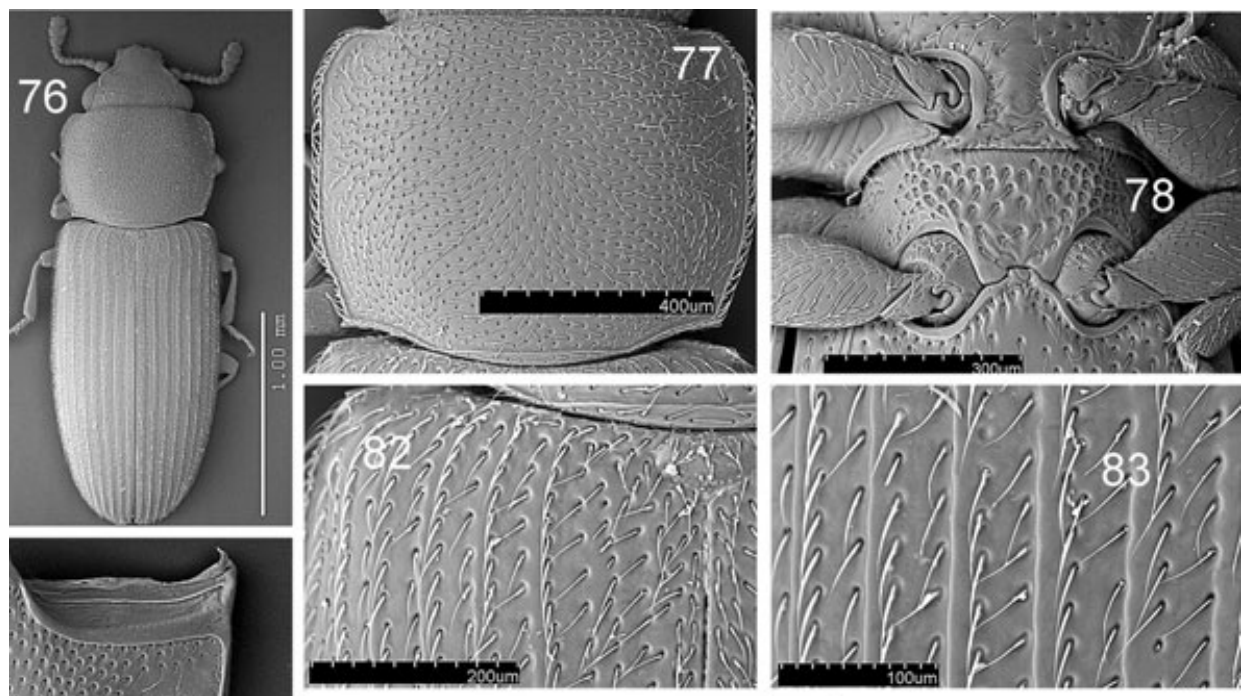
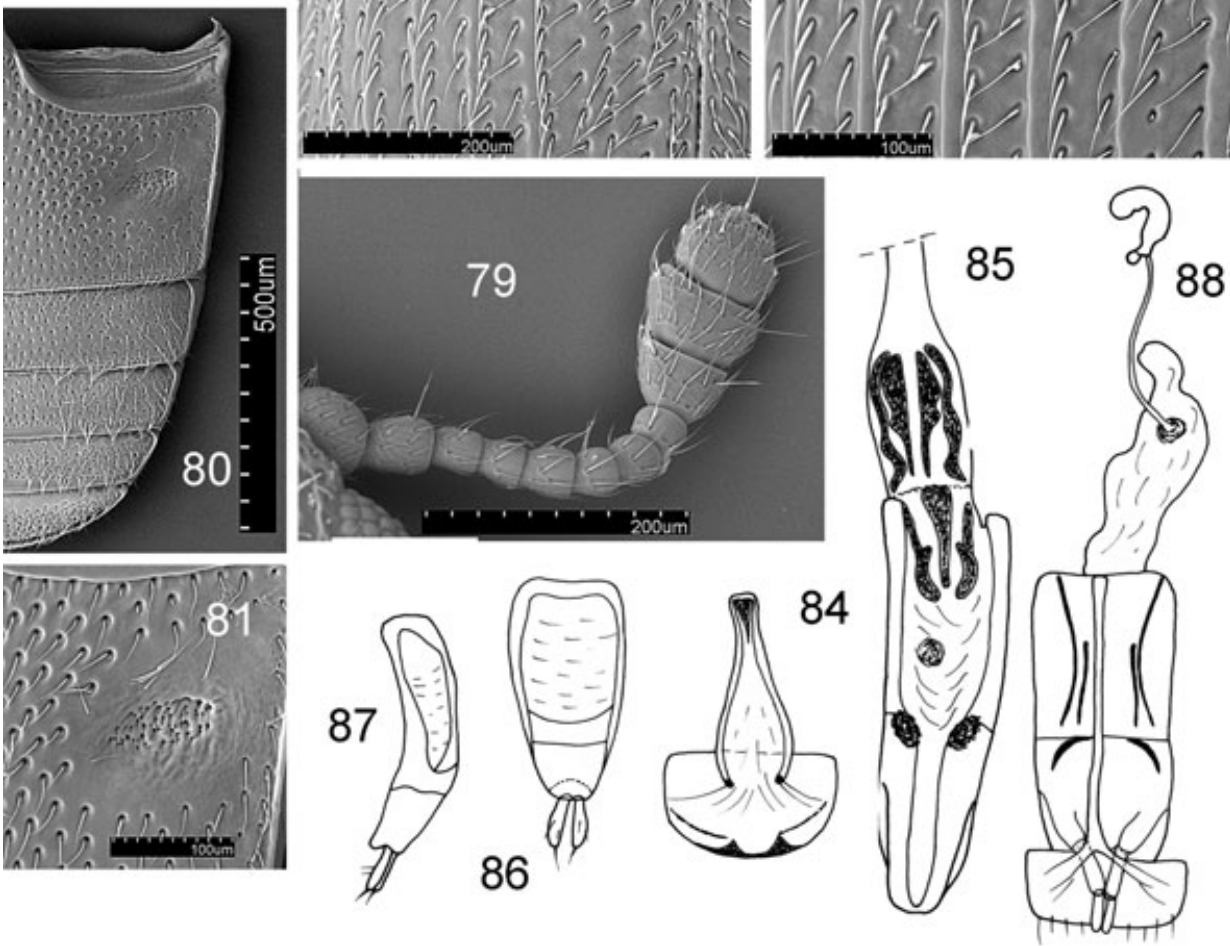

Figs 76-88. Myrabolia lawrencei: (76) dorsal view; (77) pronotum; (78) pro- and mesothorax, ventral and metaventral junction; (79) antenna; (80) male abdomen; (81) male abdominal ventrite 1, lateral part; (82) elytron, basal part; (83) elytron, disc; (84) sternite of male genital segment, ventral view; (85) median lobe; (86) tegmen, ventral; (87) tegmen, lateral; (88) female genitalia, ventral.

\section{Myrabolia lawrencei sp. n. (Figs 76-88, I 58)}

Types. Holotype, male. Australian Capital Territory. 'Black Mt. ACT, light trap, 29.iii.68. M.S. Upton' (ANIC). Paratypes: same data as holotype but, 8.iii.68 (2: ANIC); same but 26.iii.68 (1: ANIC); same but 19.iii.68 (1: ANIC); same but 13.iii.68 (1: ANIC; 1: MIZ); Black Mt. ACT, December 1987, J.F. Lawrence (1: MIZ). New South Wales. 35.30S 150.18E Kioloa SF, 15 km NE Batemans Bay, iii.1987. M.G. Robinson, flight intercept trap (1: ANIC); 35.58S 150.09E Congo, $8 \mathrm{~km} \mathrm{SE}$ by E of Moruya, 14.ii.1981, M.S. Upton (1: ANIC; 1: MIZ); same but 8.ii.1981 (1: ANIC); Dr K.K. Spence (1: ANIC; 1: MIZ); Tindabine, F.E. Wilson Collection (1: VM); Victoria. 37.28S 145.47E, Mt. Margaret Rd. at Yanks Folly Tr. $750 \mathrm{~m}$, NNE Marysville, 934, 17-28.ii.1993, A. Newton \& M. Thayer/open Eucalyptus ssp. (peppermint \& gum for.) FMHD \#93-108, ex window trap (1: ANIC).

Diagnosis. This species resembles $M$. longicornis, M. tasmanica and M. leai in having lateral margins of the pronotum distinctly sinuate, and the pronotum as wide in $1 / 4$ of apical length as in $1 / 3$ of basal length. However, antennomeres 4-8 being at most as long as wide separate $M$. lawrencei from all these species. Moreover the uniformly dark reddish-brown body can separate it from M. tasmanica and M. leai, and the body less elongate and the prosternal process truncate at apex distinguish M. lawrencei from M. longicornis.

Description. Length $2.50-2.75 \mathrm{~mm} . \quad \mathrm{BL} / \mathrm{EW}=2.90-3.02$; $\mathrm{PL} / \mathrm{PW}=0.77-0.83 ; \quad \mathrm{PL} / \mathrm{EL}=0.39-0.42 ; \quad \mathrm{PW} / \mathrm{EW}=0.88-$ $0.90 ; \mathrm{EL} / \mathrm{EW}=1.72-1.81 ; \mathrm{EL} / \mathrm{PL}=2.39-2.58$.

Body (Fig. 76) reddish-brown or sometimes light brown covered with comparatively long hairs. Antenna (Fig. 79) 0.85 times as long as pronotum with antennomeres 3 slightly longer than wide, antennomeres $4-8$ at most as long as wide with antennomere 5 slightly wider than 4 or 6 ; club long, broad and compact. Pronotum (Fig. 77) with sides distinctly sinuate, equally broad at $1 / 3$ of basal length and in $1 / 4$ of its apical length; lateral margins crenulate, with dense, sharp denticles, slightly larger along basal half; posterior angle with sharp, comparatively large tooth; punctures moderately large, $1-1.5$ 
Figs 89-95. Myrabolia australisaustralis: (89) dorsal view; (90) pronotum; (91) antenna; (92) pro- and mesothorax, ventral and metaventral junction; (93) elytron, basal part; (94) elytron, disc; (95) female genitalia, ventral.
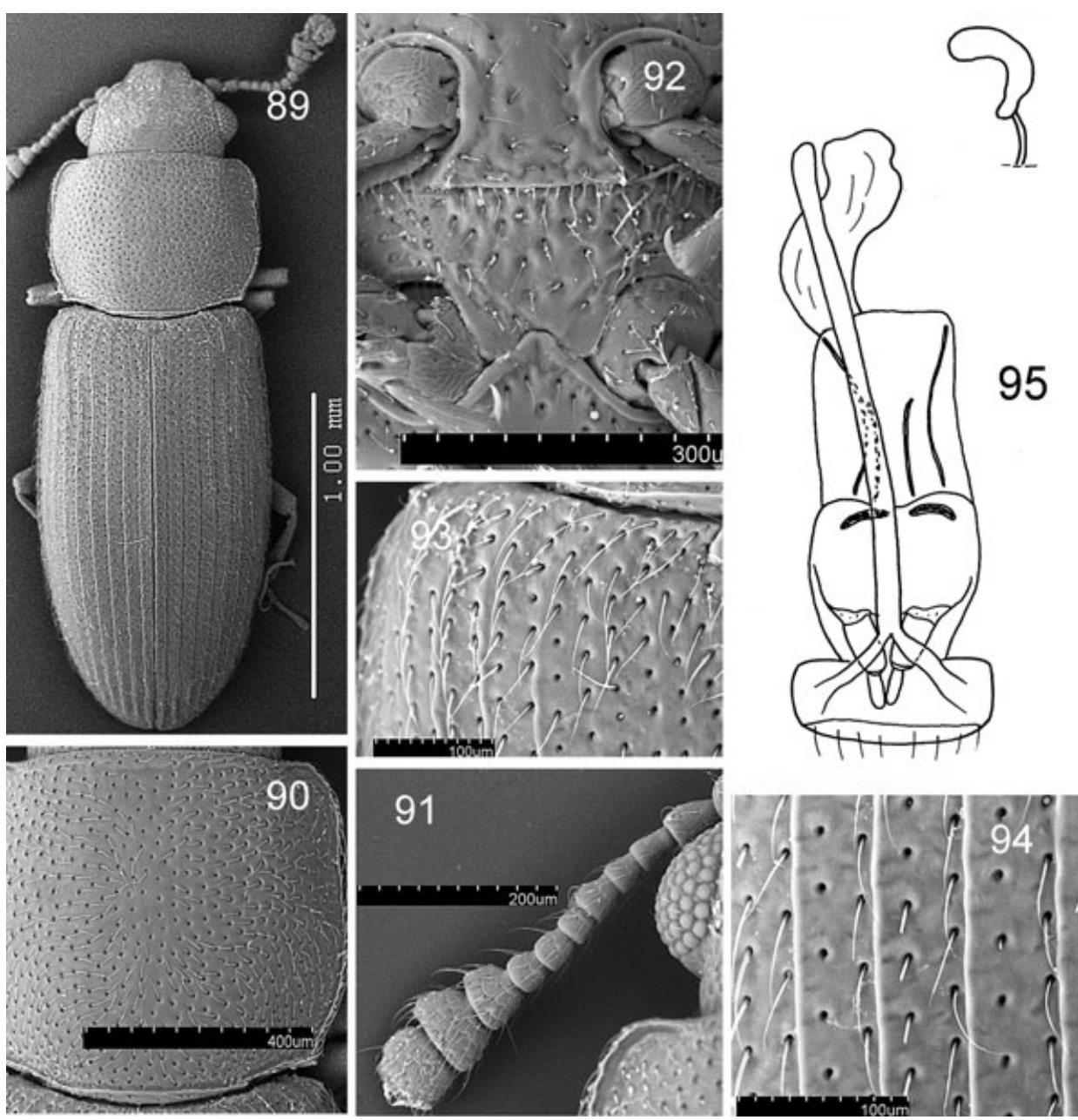

diameters apart laterally and 2-2.5 diameters apart on disc. Prosternal process (Fig. 78) punctured along lateral and apical margins; punctures rather fine and sparse; about 1.12 times as wide as coxal diameter and expanded apically, at apex about 1.65 times its width between coxae; apical margin truncate and edge somewhat crenulate. Elytra (Figs 76,82,83) with punctures in rows 1-2 diameters apart; lateral margins along shoulders moderately sharply crenulate with short, sharp tooth at anterior angle. Meso-metaventral junction covered with large punctures 0.5-1.5 diameters apart (Fig. 78); metaventrite narrowly bordered, widely and rather shallowly incised into mesoventrite (Fig. 78). Male abdominal ventrite 1 with a pair of moderately large, oval pore plates (Figs 80,81). Sternite of male genital segment and median lobe as in Figures 84 and 85. Tegmen with parameres subcylindrical, weakly curved outwardly, about $1 / 5$ as long as rest of tegmen (Figs 86,87). Female genitalia as in Figure 88.

Etymology. Named after Dr John F. Lawrence (formerly with CSIRO Entomology).

Distribution. New South Wales and Victoria (Fig. 158).

\section{Myrabolia australis sp. n. (Figs 89-95, 158 )}

Types. Holotype, female. Tasmania. 'Hobart, Lea, 10801/ parva/Myrabolia parva, Blackb., Tasmania/S. Aust. Mus. Specimen' (SAM).
Diagnosis. Myrabolia australis is most similar to M. grouvelliana but can be separated from that species in having the antennomere 5 distinctly wider than 4 or 6 , the antennal club wider and more stout, and the prosternal process truncate at apex.

Description. Length $2.36 \mathrm{~mm}$. $\quad \mathrm{BL} / \mathrm{EW}=2.96 ; \quad \mathrm{PL} / \mathrm{PW}=$ $0.82 ; \mathrm{PL} / \mathrm{EL}=0.40 ; \mathrm{EL} / \mathrm{EW}=1.79 ; \mathrm{EL} / \mathrm{PL}=2.55$.

Body (Fig. 89) elongate-oval, uniformly dark reddishbrown with moderately long hairs. Antenna (Fig. 91) about 0.95 times as long as pronotum, antennomere 3 longer than wide; antennomeres $4-8$ at most as long as wide with antennomere 5 distinctly wider than 4 or 6 ; club long, broad and compact. Pronotum (Fig. 90) with sides weakly sinuate; broadest at about $1 / 3$ of basal length; lateral margins weakly crenulate, with distinct, sharp denticles; posterior angle with small, weak tooth; punctures moderately large and dense, 1-1.5 diameters apart laterally and 2-2.5 diameters apart on disc. Prosternal process (Fig. 92) 1.33 times as wide as coxal diameter and apically 1.45 times its width between coxae; apical edge truncate. Elytra (Figs 89,93,94) with punctures in rows 2.0-3.0 diameters apart; finely crenulate along shoulders with small but distinct tooth at anterior angle. Mesometaventral junction covered with large punctures 1-1.5 diameters apart (Fig. 92); metaventrite narrowly bordered, widely but shallowly incised into mesoventrite (Fig. 92). 

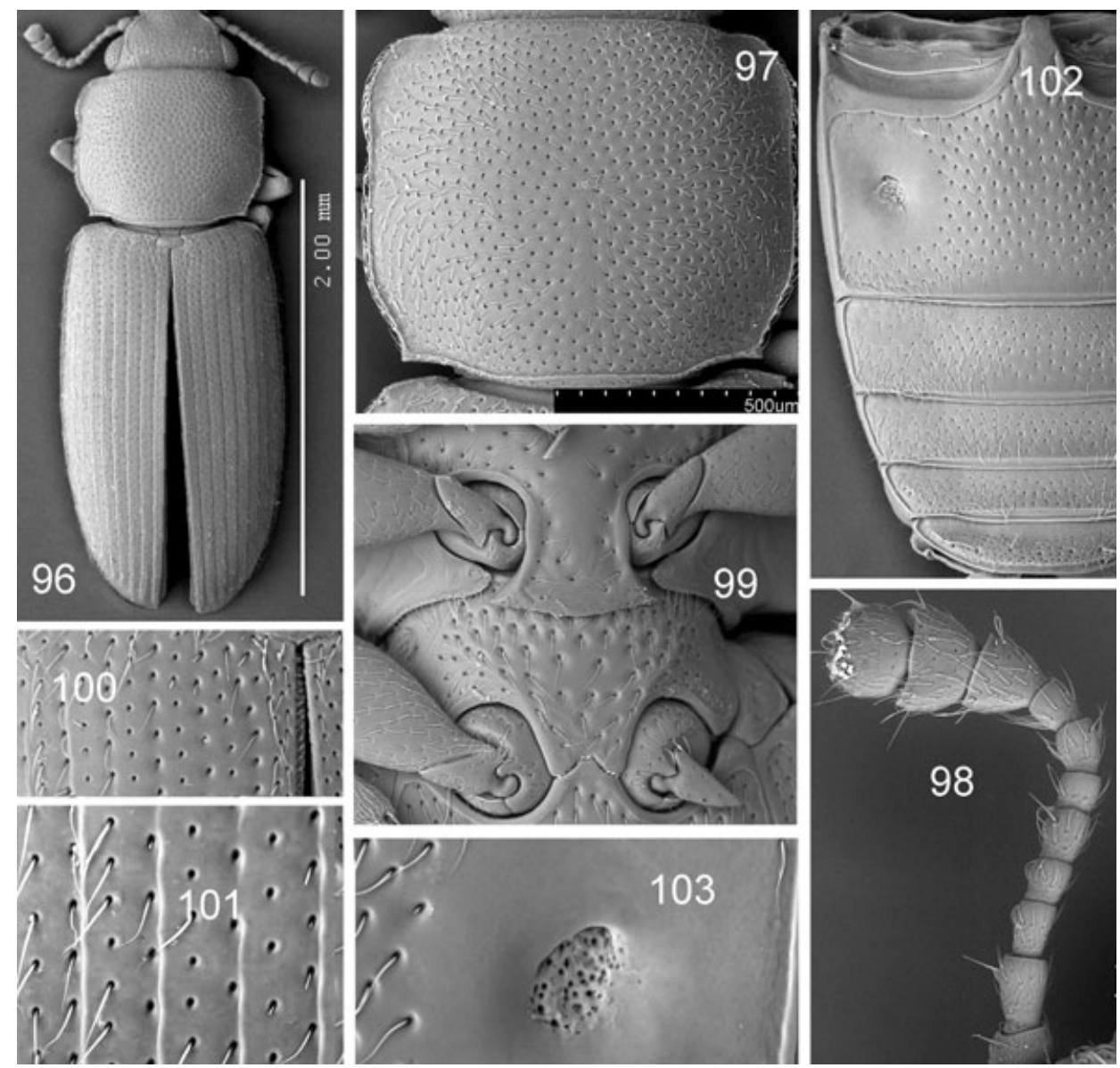

Figs 96-103. Myrabolia longicornis: (96) dorsal view; (97) pronotum; (98) antenna; (99) pro- and mesothorax, ventral and metaventral junction; (100) elytron, basal part; (101) elytron, disc; (102) male abdomen; (103) male abdominal ventrite 1 , lateral part.

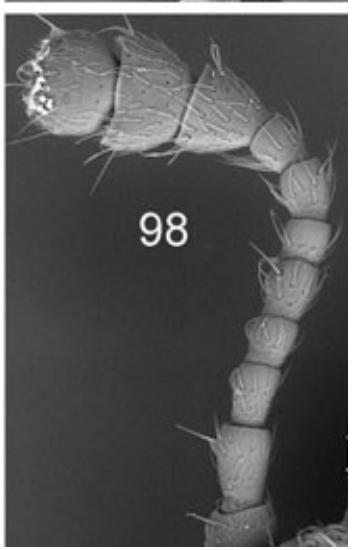

Female genitalia and shape of spermatheca as in Figure 95. Male unknown.

Etymology. Species name is formed from the Latin australis (= southern) referring to the most southern distribution of the Tasmanian species.

Distribution. Tasmania.

\section{Myrabolia longicornis Blackburn (Figs 96- 108, I 58)}

Myrabolia longicornis Blackburn 1903: 148.

Types. Lectotype, male: 'Myrabolia longicornis Blackb./ Australia, Blackburn coll., B.M. 1910-236/type, H.T./T 1216, Victoria' (BMNH). Paralectotype, female: 'Myrabolia longicornis cotype/28774 Myrabolia longicornis B1. Vic. Tas. Cotype/Victoria Blackburn' (SAM). Present designation. The lectotype is designated to stabilise the taxonomic status of this species.

Other specimens examined. Victoria. Belgrave, F.E. Wilson, September 1936 (1: VM; 1: MIZ); Dividing Range, Blackburn (1: SAM; 1, ANIC).

Diagnosis. This species resembles $M$. lawrencei, $M$. tasmanica and $M$. leai in the shape of pronotum with its lateral margins distinctly sinuate, and is equally wide in $1 / 4$ of apical and $1 / 3$ of basal length. It can be distinguished from these species by uniformly chestnut-brown (rarely yellowish)
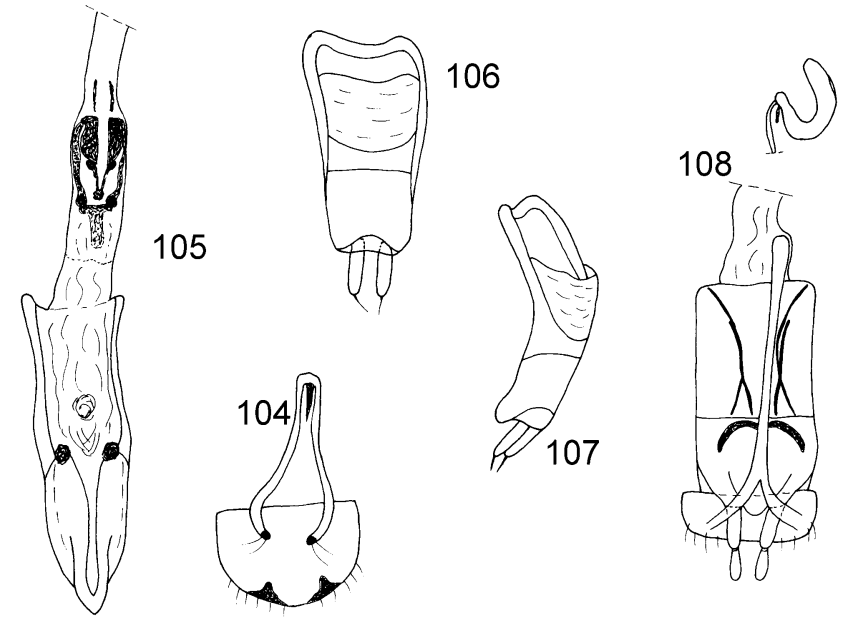

Figs 104-108. Myrabolia longicornis: (104) sternite of male genital segment, ventral view; (105) median lobe; (106) tegmen, ventral; (107) tegmen, lateral; (108) female genitalia, ventral.

colour, strongly elongate body and elytra, the antennomeres 3-5 and 7-8 at least slightly longer than wide and prosternal process widely rounded at apex.

Description. Length $2.53-3.27 \mathrm{~mm}$. BL/EW = 3.06-3.16; $\mathrm{PL} / \mathrm{PW}=0.88-0.89 ; \quad \mathrm{PL} / \mathrm{EL}=0.41-0.43 ; \quad \mathrm{PW} / \mathrm{EW}=0.87-$ $0.92 ; \mathrm{EL} / \mathrm{EW}=1.86-1.90 ; \mathrm{EL} / \mathrm{PL}=2.30-2.45$. 
Figs 109-115. Myrabolia blackburni: (109) pronotum; (110) pro- and mesothorax, ventral and metaventral junction; (111) antenna; (112) elytron, basal part; (113) elytron, disc; (114) male abdomen; (115) male abdominal ventrite 1 , lateral part.
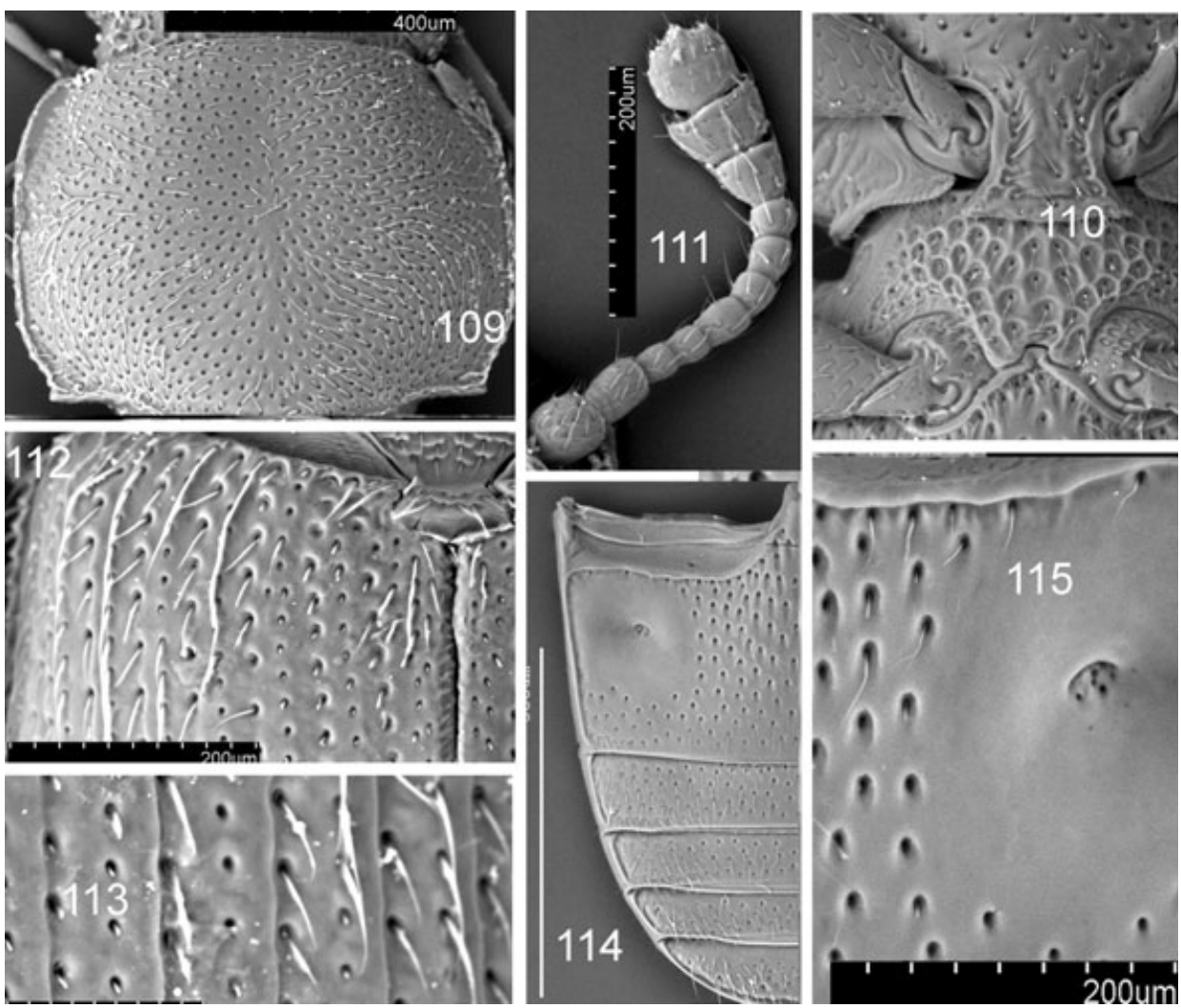

Body (Fig. 96) long-oval, chestnut-brown or sometimes yellowish-brown, covered with comparatively long hairs. Antenna (Fig. 98) 0.9 times as long as pronotum with antennomeres $3-5$ and $7-8$ at least slightly longer than wide; antennomere 4 weakly elongate, shorter than 3 or 5 ; antennomere 6 shorter than wide; club long, broad and rather compact. Pronotum (Fig. 97) with sides sinuate, widened near anterior angle and in 1/3 of basal length; lateral margins crenulate, with moderately large denticles, larger along basal half; posterior angle with sharp bearing comparatively large tooth; punctures moderately large, 2-3 diameters apart. Prosternal process (Fig. 99) finely and sparsely punctured, about 1.2 times as broad as coxal diameter and apically 1.6 times its width between coxae; with apical margin widely rounded. Elytra (Figs 96,100,101) with punctures in rows 2-3 diameters apart; lateral margins along shoulders weakly crenulate. Meso-metaventral junction with moderately large punctures 1.5-2.0 diameters apart (Fig. 99); metaventrite not bordered and rather shallowly incised into mesoventrite (Fig. 99). Male abdominal ventrite 1 with a pair of moderately large, obliquely oval pore plates (Figs 102,103). Sternite of male genital segment and median lobe as in Figures 104 and 105. Tegmen with parameres cylindrical, about $1 / 5$ as long as rest of tegmen (Figs 106,107). Female genitalia as in Figure 108.

Distribution. Known from few localities in Victoria (Fig. 158).
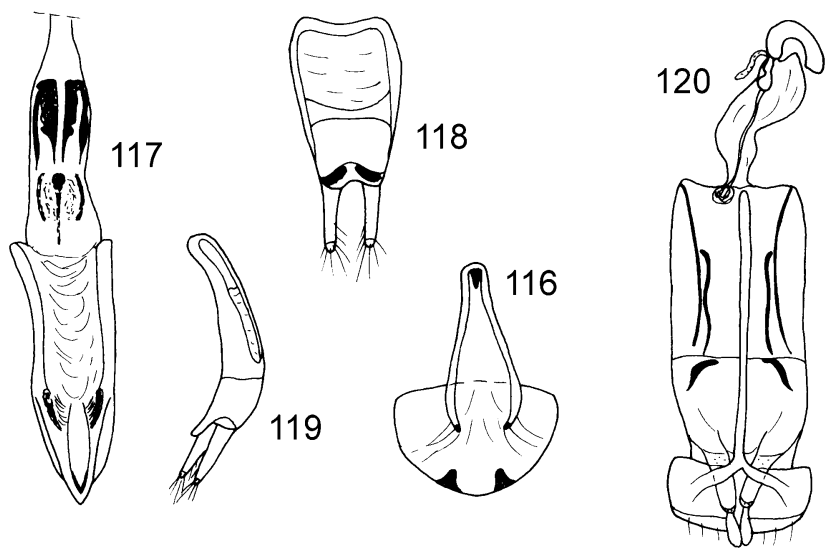

Figs 116-120. Myrabolia blackburni: (116) sternite of male genital segment, ventral view; (117) median lobe; (118) tegmen, ventral; (119) tegmen, lateral; (120) female genitalia, ventral.

\section{Myrabolia blackburni sp. n. (Figs I09-I 20, I58)}

Types. Holotype, male. South Australia. 'Tumby Bay, S. Australia, Blackburn/Myrabolia lindensis, cotype/Myrabolia lindensis Bl., cotype, 9.8775' (SAM). Paratypes: female, same data as holotype (SAM); S.A. Yumali, 8.viii.1968, Colles \& Liepa (1 male, dissected: MIZ).

Note. The holotype and one paratype belonged to Blackburn's type series of $M$. lindensis.

Diagnosis. This species resembles $M$. lindensis in a body shape and colouration. However, it is smaller and has 

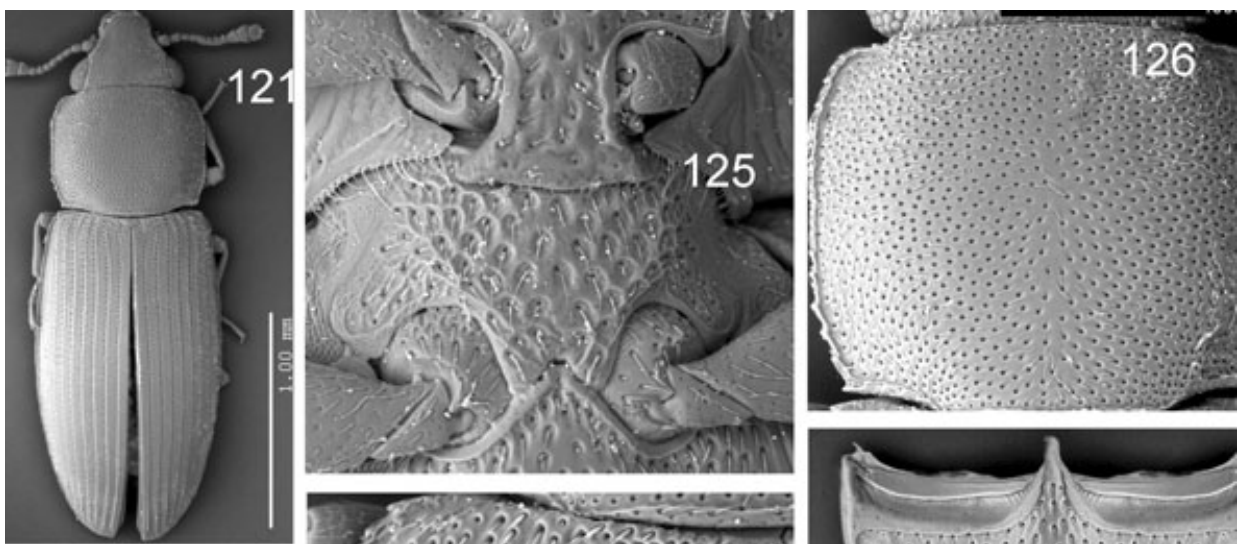

Figs 121-128. Myrabolia lindensis: (121) dorsal view; (122) antenna; (123) male abdomen; (124) male abdominal ventrite 1, lateral part; (125) pro- and mesothorax, ventral and metaventral junction; (126) pronotum; (127) elytron, basal part; (128) elytron, disc.
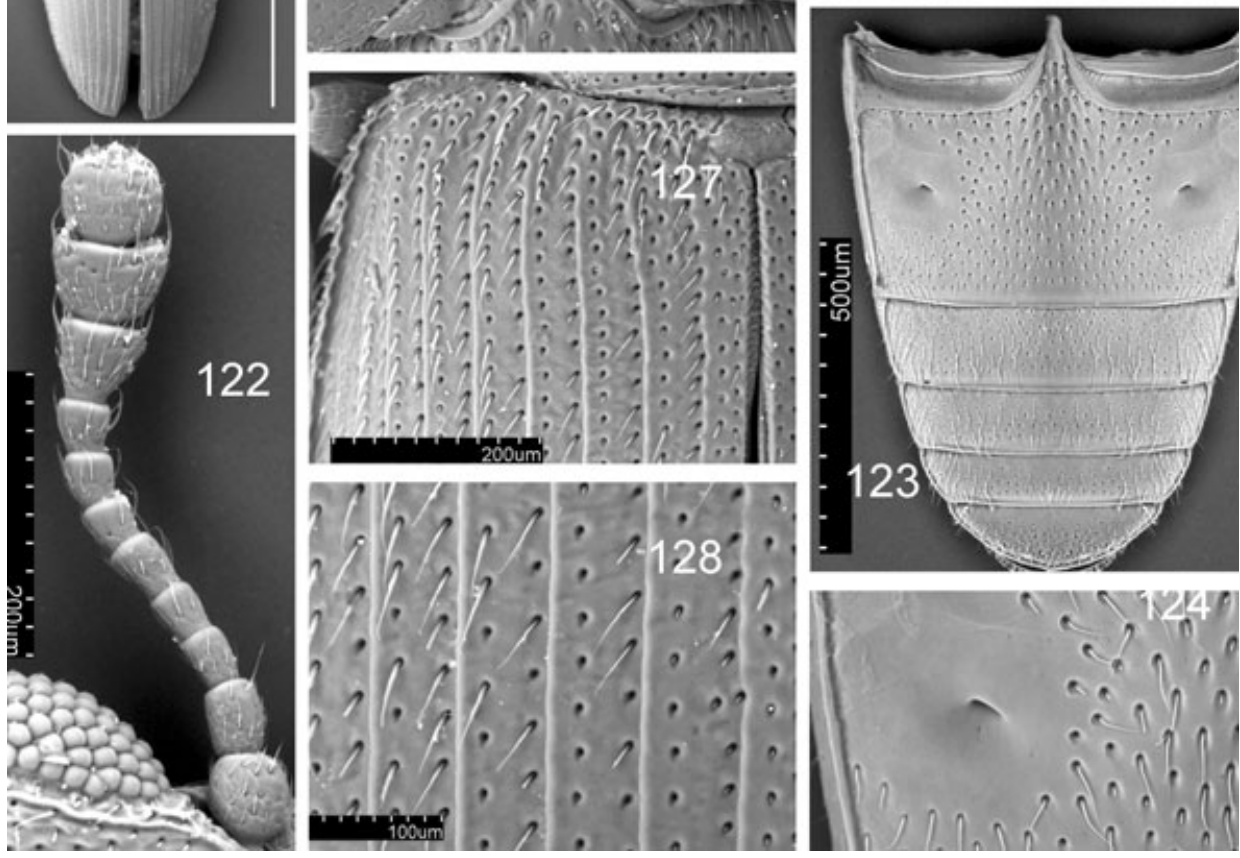

proportionally shorter body with shorter pronotum and elytra. The shape of antennomeres 3-4 that are at most as long as wide, mesoventral process with very large punctures, male abdominal ventrite 1 with a pair of small pore plates (not pits), and tegmen with much longer parameres separate easily $M$. blackburni from $M$. lindensis.

Description. Length $2.15-2.25 \mathrm{~mm} . \quad \mathrm{BL} / \mathrm{EW}=2.88-2.91$; $\mathrm{PL} / \mathrm{PW}=0.76-0.79 ; \quad \mathrm{PL} / \mathrm{EL}=0.38-0.41 ; \quad \mathrm{PW} / \mathrm{EW}=0.85-$ $0.87 ; \mathrm{EL} / \mathrm{EW}=1.68-1.71 ; \mathrm{EL} / \mathrm{PL}=2.43-2.60$.

Body elongate-oval, yellowish-brown, covered with comparatively short hairs. Antenna (Fig. 111) 0.8 times as long as pronotum with antennomeres 3-4 and 6-8 at most as long as wide; antennomere 5 distinctly longer than broad; club long, moderately broad and compact. Pronotum (Fig. 109) with sides finely rounded towards anterior and posterior margins; widest at about $1 / 4$ of basal length; lateral margins weakly crenulate, with small denticles, slightly larger near base; posterior angle with sharp tooth; punctures moderately large, 1-1.5 diameters apart laterally and 1.5-2 diameters apart on disc. Prosternal process (Fig. 110) coarsely punctured along lateral margins, about 1.25 times as wide as coxal diameter, moderately strongly expanded apically and at apex 1.5 times its width between coxae; apical margin weakly sinuate. Elytra (Figs 112,113) with punctures in rows 2.0-2.5 diam- eters apart; lateral margins along shoulders sharply crenulate, with tooth at anterior angle as large as teeth below it. Mesometaventral junction covered with very large punctures almost touching each other (Fig. 110); metaventrite narrowly bordered and rather shallowly incised into mesoventrite (Fig. 110). Male abdominal ventrite 1 with a pair of very small, oval pore plates (Figs 114,115). Sternite of male genital segment and median lobe as in Figures 116 and 117. Tegmen with parameres very long, tapering towards apex, about $1 / 3$ as long as rest of tegmen (Figs 118,119). Female genitalia as in Figure 120.

Etymology. This new species is named after T. Blackburn who attempted to understand this difficult genus of beetles.

Distribution. South Australia (Fig. 158).

\section{Myrabolia lindensis Blackburn (Figs I $\mid$ I-I32, I 58)}

Myrabolia lindensis Blackburn 1892: 31.

Types. Lectotype, male. South Australia. 'Myrabolia lindensis Blackb./Australia, Blackburn coll., B.M. 1910-236/type, H.T.' (BMNH). Paralectotype, female: 'Myrabolia lindensis B1. cotype/15 590, S. Australia (SAM). 

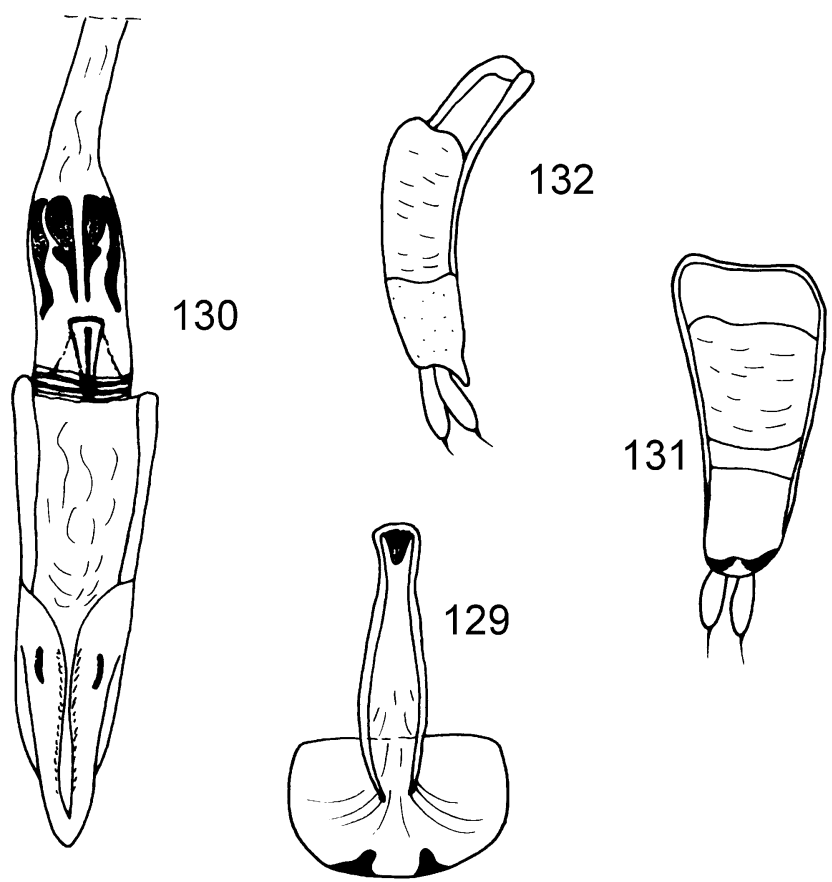

Figs 129-132. Myrabolia lindensis: (129) sternite of male genital segment, ventral view; (130) median lobe; (131) tegmen, ventral; (132) tegmen, lateral.

Present designation. The lectotype of Myrabolia lindensis Blackburn 1892 is made to fix the taxonomic status of this species.

Other specimens examined. New South Wales. $32 \mathrm{~km} \mathrm{~S}$ of Willston, 17.iv.1977, B.B. Lowery (1: ANIC); Victoria. $7.3 \mathrm{~km}$ SW of Wemen (34.50S 142.34E), 25.x.-3.xi.1988, T. Weir, J. Lawrence \& M. Hansen; at night mallee (1: MIZ).

Diagnosis. This species is most similar to M. blackburni and M. grouvelliana but can be separated from both of these species by longer and less stout antennae with antennomeres 3-5 distinctly longer than broad and the male abdominal ventrite 1 provided with a pair of very small, oval pits.

Description. Length $2.23-2.56 \mathrm{~mm}$. $\quad \mathrm{BL} / \mathrm{EW}=2.93-2.96$; $\mathrm{PL} / \mathrm{PW}=0.79-0.83 ; \quad \mathrm{PL} / \mathrm{EL}=0.38-0.41 ; \quad \mathrm{PW} / \mathrm{EW}=0.86-$ $0.92 ; \mathrm{EL} / \mathrm{EW}=1.76-1.80 ; \mathrm{EL} / \mathrm{PL}=2.45-2.55$.

Body (Fig. 121) elongate-oval, dark yellowish-brown, covered with comparatively short hairs. Antenna (Fig. 122) about 0.9 times as long as pronotum with antennomeres 3-5 distinctly longer than wide and antennomeres 6-8 at most as long as wide; antennomere 4 as long as 3 and distinctly shorter than 5; club long, moderately broad and rather compact. Pronotum (Fig. 126) with sides almost straight along middle length, finely rounded towards anterior and posterior margins; lateral margins crenulate, with sharp denticles, larger near base; posterior angle with sharp, comparatively large tooth; punctures moderately large, 1-1.5 diameters apart laterally and 2 diameters apart on disc. Prosternal process (Fig. 125) coarsely and moderately densely punctured, about as wide as coxal diameter, strongly widened at apex (at apex 1.7 times its width between coxae) with apical margin weakly sinuate.
Elytra (Figs 121,127,128) with punctures in rows 1.5-2.0 diameters apart; lateral margins along shoulders sharply crenulate, with moderately large tooth at anterior angle. Mesometaventral junction covered with large punctures $0.5-1.0$ diameter apart (Fig. 125); metaventrite narrowly bordered and moderately deeply incised into mesoventrite (Fig. 125). Male abdominal ventrite 1 with a pair of very small, oval pits (Figs 123,124). Sternite of male genital segment and median lobe as in Figures 129 and 130. Tegmen with parameres subcylindrical, about $1 / 5$ as long as rest of tegmen (Figs 131,132). Female genitalia not studied.

Distribution. South Australia, Victoria, New South Wales (Fig. 158).

\section{Myrabolia leai (Grouvelle) comb. $n$. (Figs I33-I44, I 58)}

Ocholissa leai Grouvelle 1911: 135.

Types. Holotype, female. Tasmania, Hobart, 'Lea/Lea 13678/type/voisin Ocholissa/Ocholissa leae Grouv. 5'. (MNHN).

Other specimens examined. New South Wales. Sydney, Lea (1: ANIC); Kioloa SF $15 \mathrm{~km} \mathrm{NE} \mathrm{Batemans} \mathrm{Bay} \mathrm{(35.30S}$ 150.18E), iv.1987, M.G. Robinson, ex sticky trap (1: ANIC); same but v.1987 (1: ANIC); Forest Reefs, Lea (1: SAM); $50 \mathrm{~km}$ E of Coonabarabran, 29.iii.1980, G. Monteith (2: QM). Australian Capital Territory. Piccadilly Circus (35.22S 148.48E), $1240 \mathrm{~m}$ ii.1984, J. Lawrence, T. Weir, M.-L. Johnson, flight intercept window/trough trap (1: ANIC); Wombat Ck. (35.19S 148.51E), 6 km. NE of Piccadilly Circus, 750 m ACT, ix.1984, Weir, Lawrence, Johnson (1: ANIC; 1: MIZ). Victoria. Baw Baw Alpine Res (37.50S 146.16E), $c$. $1 \mathrm{~km}$ WNW Alpine Village, 1420 m, A. Newton, M. Thayer, under bark, flakes live Eucalyptus pauciflora (1: MIZ); Mt Buffalo, Blackburn (1: SAM). South Australia. Deep Creek Cons. Park (35.37S 138.16E), 19-20.iii.1981, E. Matthews \& J. Forrest (2: SAM); 14 km WNW Renmark (34.07S 140.37E), 8-21.viii.1995, A. Lambie (1: ANIC). Tasmania. Hobart, J.J. Walker (1: BMNH); Hobart; 91-88 (3: BMNH; 2: MIZ); Pelion Gap (41.52S 146.03E), $2 \mathrm{~km}$ ENE, Mt. Ossa, 12-15.ii.1991, 1120 m, under bark, A. Calder, W. Dressler (1: ANIC; 1 totally dissected: MIZ); same but 30.xi.-8.i.1991, E. Nielsen, E. Edwards, Malaise \#6, Eucalyptus coccifera woodland with alpine heath (1: ANIC); Claytons (43.22S 146.08E), Bathurst Harbour, 3.xii.1990-15.i.1991, Nielsen, Edwards, Malaise trap (1: MIZ); Mt Wellington, Lea (6: SAM). Western Australia. Donnybrook, Lea (1: SAM).

Diagnosis. This species closely resembles $M$. tasmanica in having the body more or less distinctly bicoloured (brown and black). However, M. leai is distinguished by shorter pronotum and elytra and the male abdominal ventrite 1 with a pair of rounded pore plate of moderate size.

Description. Length $2.10-2.56 \mathrm{~mm}$. BL/EW = 2.84-3.00; $\mathrm{PL} / \mathrm{PW}=0.81-0.84 ; \quad \mathrm{PL} / \mathrm{EL}=0.38-0.39 ; \quad \mathrm{PW} / \mathrm{EW}=0.83-$ $0.86 ; \mathrm{EL} / \mathrm{EW}=1.71-1.80 ; \mathrm{EL} / \mathrm{PL}=2.50-2.63$.

Body (Figs 133,134) yellowish-brown to dark-brown with entire pronotum, transverse band across middle of elytra, 

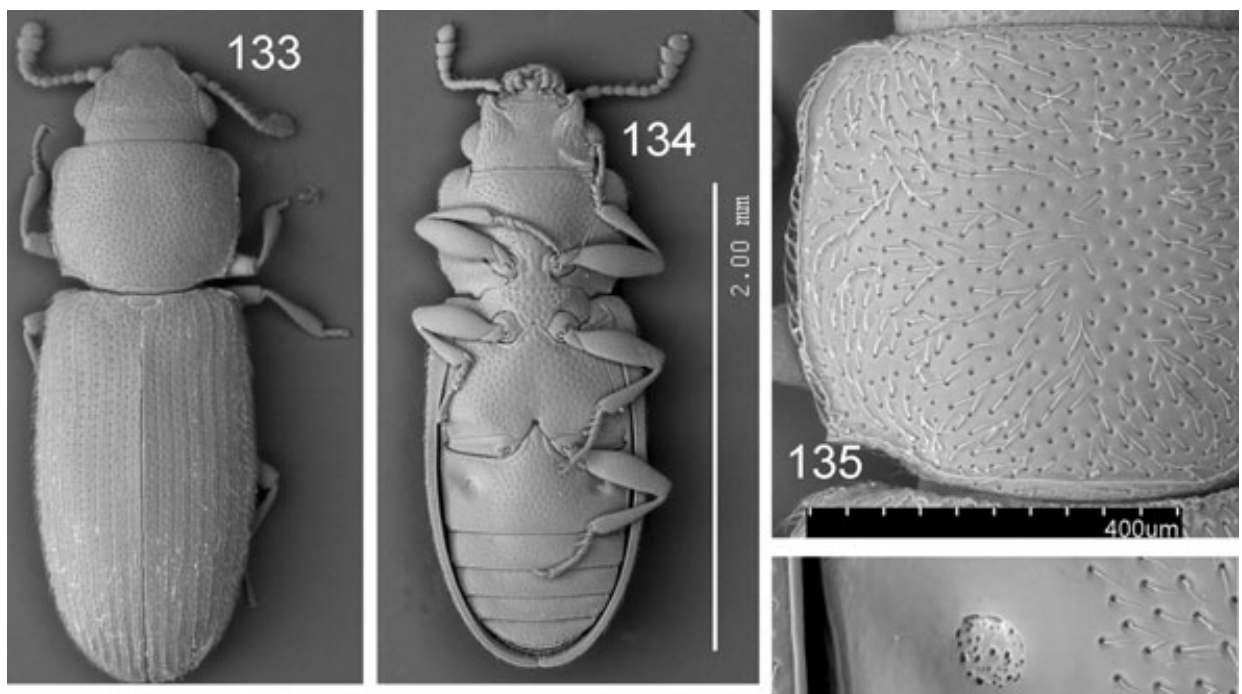

Figs 133-139. Myrabolia leai: (133) dorsal view; (134) ventral view; (135) pronotum; (136) pro- and mesothorax, ventral and metaventral junction; (137) antenna; (138) male abdomen; (139) male abdominal ventrite 1 , lateral part.
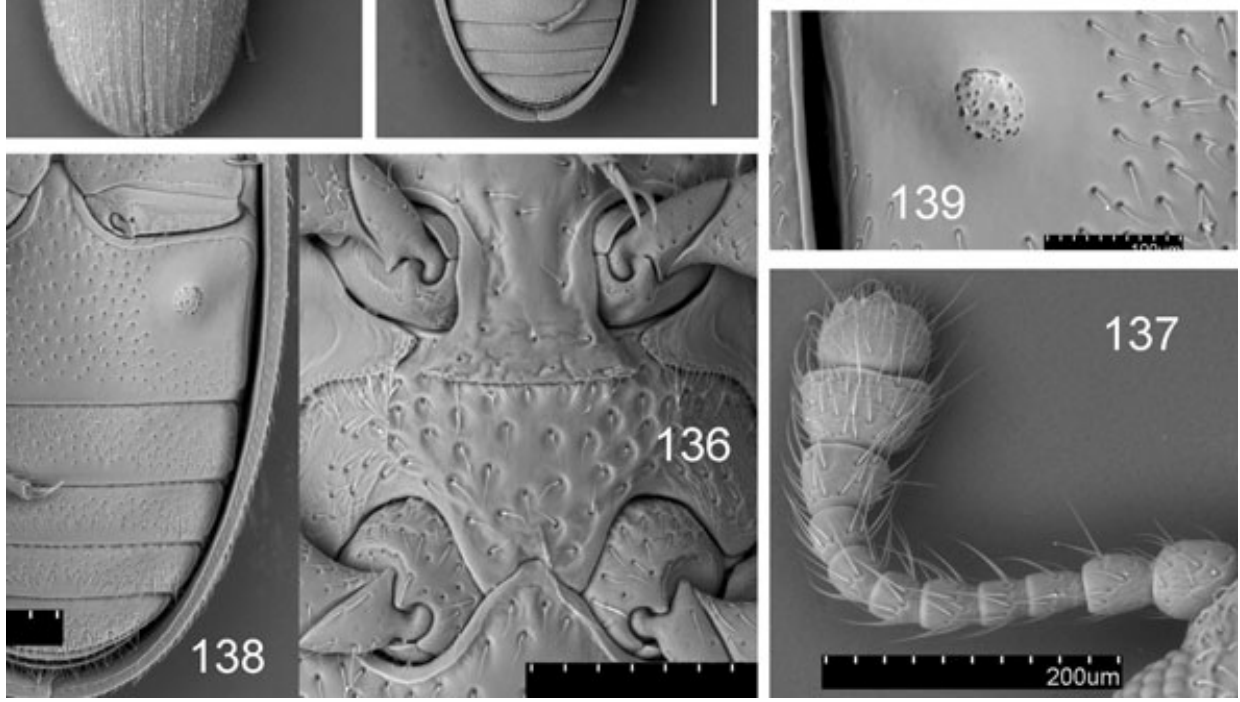

142

141
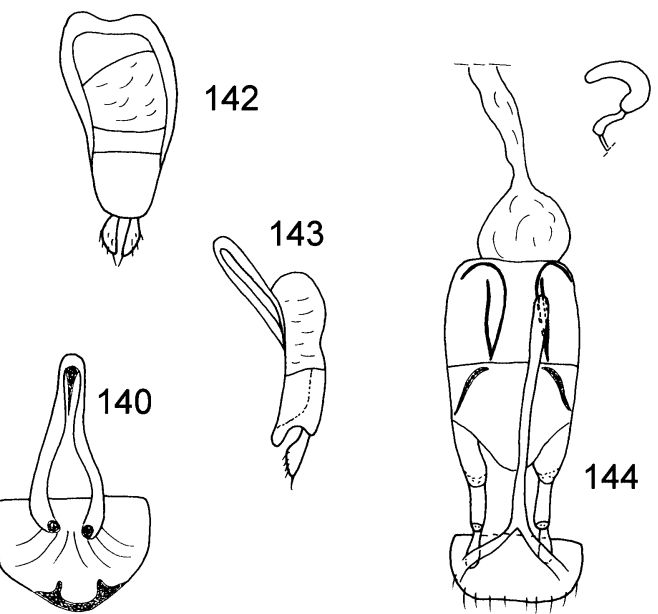

Figs 140-144. Myrabolia leai: (140) sternite of male genital segment, ventral view; (141) median lobe; (142) tegmen, ventral; (143) tegmen, lateral; (144) female genitalia.

pro-, meso- and metaventrite brownish-black to black. Antenna (Fig. 137) 0.9 times as long as pronotum with antennomeres 3-5 longer than wide, but antennomere 4 slightly shorter than 3 or 5 ; antennomeres $6-8$ at most as

Journal compilation (C) 2008 Australian Entomological Society long as wide; club long, broad and rather compact. Pronotum (Fig. 135) with sides somewhat sinuate, narrowing at $1 / 3$ length from anterior margin and near base; widest at about $1 / 3$ of basal length and 1/4 of apical length; lateral margins weakly crenulate with very small denticles; posterior angle with distinct tooth; punctures moderately large and almost as dense laterally as on disc, 2-3 diameters apart. Prosternal process (Fig. 136) very finely and sparsely punctured, about 1.1 times as wide as coxal diameter and at apex 1.5 times width between coxae; with apical margin truncate and with edge somewhat crenulate. Elytra (Fig. 133) with punctures in rows 2.0-2.5 diameters apart; lateral margins along shoulders sharply crenulate, with distinct tooth at anterior angle. Mesometaventral junction covered with punctures 1.0-2.0 diameters apart (Fig. 136); metaventrite narrowly bordered and rather shallowly incised into mesoventrite (Fig. 136). Male abdominal ventrite 1 with a pair of round pore plates of moderate size (Figs 138,139). Sternite of male genital segment and median lobe as in Figures 140 and 141. Tegmen with parameres curved outwardly, about $1 / 5$ as long as rest of tegmen, shortly setose (Figs 142,143). Female genitalia as in Figure 144.

Distribution. Known from Tasmania, Victoria, New South Wales and southern part of Western Australia (Fig. 158). 
Figs 145-152. Myrabolia tasmanica: (145) dorsal view; (146) antenna; (147) pronotum; (148) pro- and mesothorax, ventral and metaventral junction; (149) elytron, disc; (150) elytron, basal part; (151) male abdomen; (152) male abdominal ventrite 1 , lateral part.
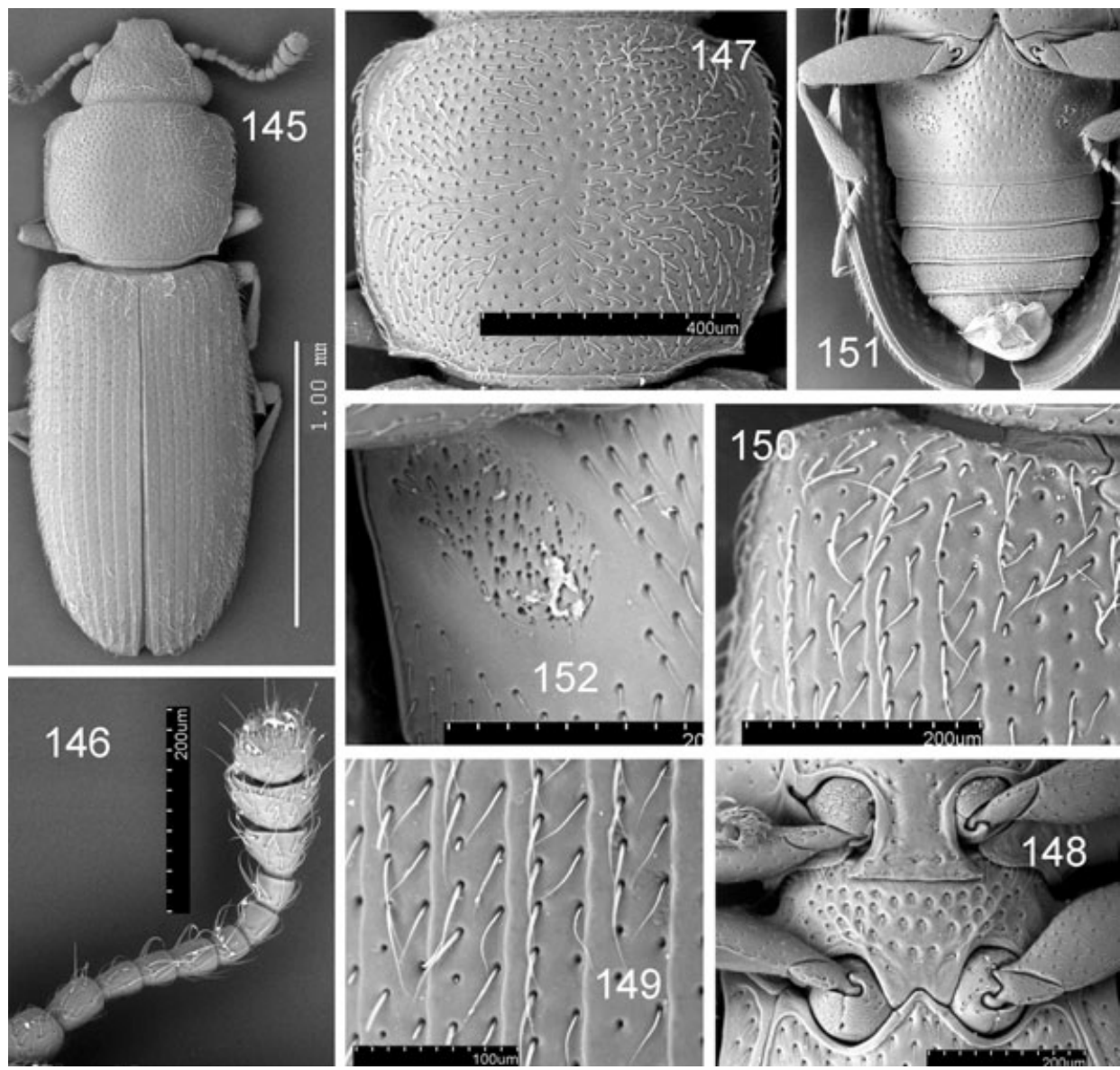
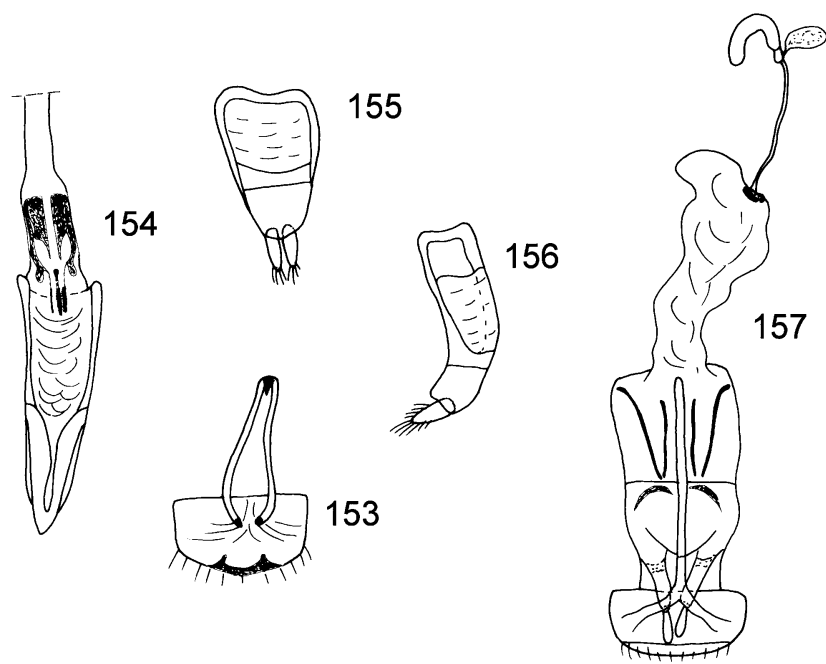

Figs 153-157. Myrabolia tasmanica: (153) sternite of male genital segment, ventral view; (154) median lobe; (155) tegmen, ventral; (156) tegmen, lateral; (157) female genitalia, ventral.

\section{Myrabolia tasmanica sp. n. (Figs I45-I58)}

Types. Holotype, male. Tasmania. 'Hobart, Lea, 10560/ longicornis/S. Aust. Mus. specimen' (SAM). Paratypes: King
I., Lea/Myrabolia longicornis B1. 10800 (1 female: SAM; 1 male MIZ); Claytons (43.22S 146.06E), Bathurst Harbour, 15 January-20 February 1991, A. Calder, W. Dressler, Malaise \#3 closed forest/ANIC database no. 25030021 (1 male, teneral: ANIC); Claytons (43.22S 146.08E), Bathurst Harbour, 15 January 1991, E. Nielsen, E. Edwards, Malaise \#3 closed forest/ANIC database no. 25030022 (1 male, teneral, totally dissected: MIZ).

Diagnosis. This species resembles M. leai in the body colouration but can be distinguished from it by having the elytra more elongate, the pronotum longer and the male abdominal ventrite 1 with a pair of large, oval pore plates.

Description. Length $2.18-2.76 \mathrm{~mm} . \quad \mathrm{BL} / \mathrm{EW}=2.91-3.05$; $\mathrm{PL} / \mathrm{PW}=0.83-0.88 ; \quad \mathrm{PL} / \mathrm{EL}=0.40-0.42 ; \quad \mathrm{PW} / \mathrm{EW}=0.87-$ $0.89 ; \mathrm{EL} / \mathrm{EW}=1.80-1.84 ; \mathrm{EL} / \mathrm{PL}=2.40-2.50$.

Body (Fig. 145) dark-brown (two yellow specimen studied were apparently teneral) with pronotum darker, blackishbrown; elytra usually with transverse, more or less distinct band across middle, pro-, meso- and metasternum blackishbrown. Antenna (Fig. 146) 0.9 times as long as pronotum with antennomeres 4 and 6-8 at most as long as wide; antennomeres 3 and 5 distinctly longer than broad and subequal in length to each other; club long, broad and rather compact. Pronotum (Fig. 147) with sides sinuate, narrowing at $1 / 3$ length from anterior margin and near base; widest at about $1 / 3$ of basal length and $1 / 4$ of apical length; lateral margins weakly 


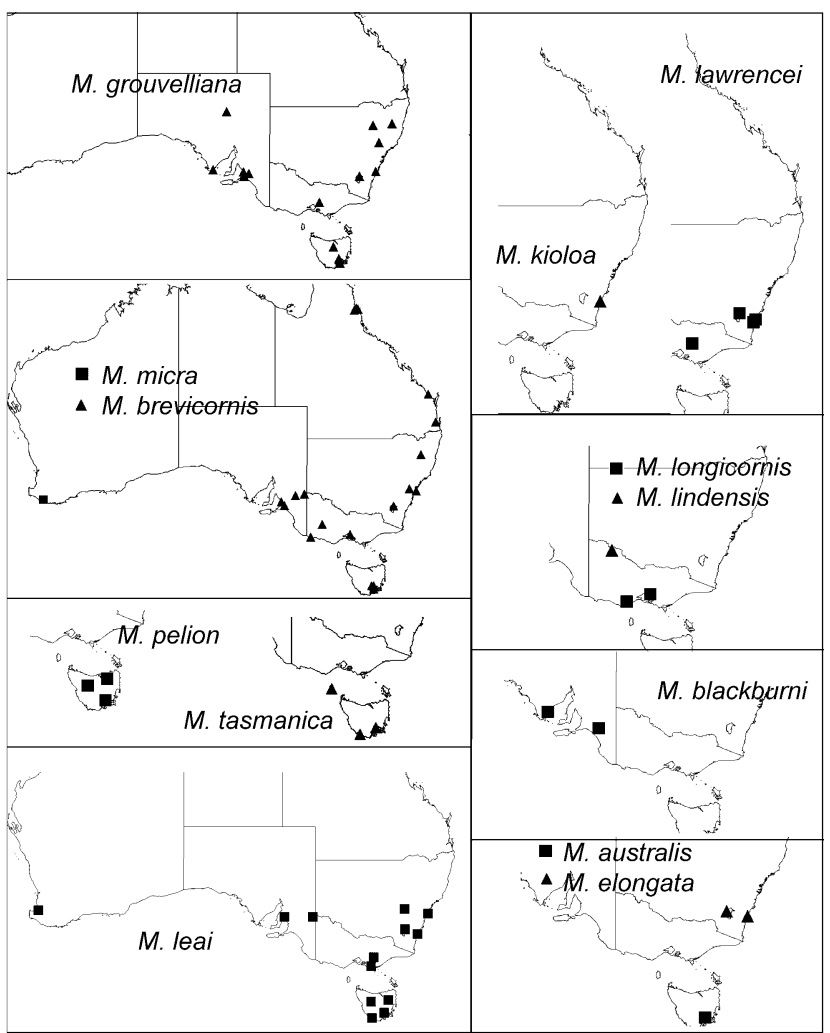

Fig. 158. Distribution maps for Myrabolia species.

crenulate, with very small denticles; posterior angle with distinct tooth; punctures moderately large, 1-1.5 diameter apart laterally and 2-2.5 diameters apart on disc. Prosternal process (Fig. 148) punctured only along lateral and apical margins; punctures sparse and fine; about 1.15 times as wide as coxal diameter, strongly expanded apically (at apex 1.7 times width between coxae) with apical margin truncate. Elytra (Figs 145,149,150) with punctures in rows 2.0-2.5 diameters apart; lateral margins along shoulders weakly crenulate, with small tooth at anterior angle. Meso- and metaventral processes covered with large punctures 1.0-1.5 diameters apart (Fig. 148); metaventrite narrowly bordered and moderately deeply incised into mesoventrite (Fig. 148). Male abdominal ventrite 1 with a pair of very large, obliquely oval pore plates (Figs 151,152). Sternite of male genital segment and median lobe as in Figures 153 and 154. Tegmen with parameres somewhat tapering towards apex, about $1 / 4$ as long as rest of tegmen, shortly setose (Figs 155,156). Female genitalia as in Figure 157.

Etymology. This new species is named after the State of its origin.

Distribution. Tasmania (Fig. 158).

\section{ACKNOWLEDGEMENTS}

We thank very much Max Barclay (BMNH), Thierry Deuve and Azadeh Taghavian (MNHN), Jan Forrest and Eric Matthews (SAM), Ottó Merkl (HNHM); Geoff Monteith (QM) Manfred Uhlig (NMB) and Ken Walker (MV) for the loan of types, and other specimens used in this study. We sincerely thank Dr Magdalena Kowalewska (MIZ) for SEM photography.

\section{REFERENCES}

Blackburn T. 1892. Further notes on Australian Coleoptera, with descriptions of new genera and species. Transactions of the Royal Society of South Australia 15, 20-73.

Blackburn T. 1903. Further notes on Australian Coleoptera, with descriptions of new genera and species. Transactions of the Royal Society of South Australia 28, 91-182.

Erichson WF. 1842. Beitrag zur Insecten - Fauna von Vandiemensland, mit besonderer Berucksichtigung der geographischen Verbreitung der Insecten. Archiv für Naturgeschichte 8, 83-287, pl. 4-5.

Grouvelle A. 1911. Descriptions de quelques nouveaux especes de Coleopteres Australiennes. Proceedings of the Royal Society of Victoria 24, 135-136.

Lawrence JF. 1988. Notes on the classification of some Australian Cucujoidea. Journal of the Australian Entomological Society 27, 53-54.

Lawrence JF \& Britton EB. 1991. Coleoptera (beetles). In: Insects of Australia. A Textbook for Students and Research Workers, 2nd edn, Vol. 2 (ed. CSIRO Division of Entomology), pp. 543-683. Melbourne University Press, Carlton, Victoria, Australia.

Leschen RAB, Lawrence JF \& Slipinski SA. 2005. Classification of basal Cucujoidea (Coleoptera: Polyphaga): cladistic analysis, keys and review of new families. Invertebrate Systematics 19, 17-73.

Reitter E. 1876. Neue Gattungen und Arten aus der Familie der Cucujidae. Coleopterologische Hefte 15, 35-64.

Reitter E. 1878. Coleopterorum species novae. Verhandlungen der Zoologisch-Botanischen Gesellschaft in Wien 27, 165-194.

SenGupta T \& Crowson RA. 1969. Further observations on the family Boganiidae, with definition of two new families Cavognathidae and Phloeostichidae. Journal of Natural History 3, 571-590.

Accepted for publication 23 June 2007. 